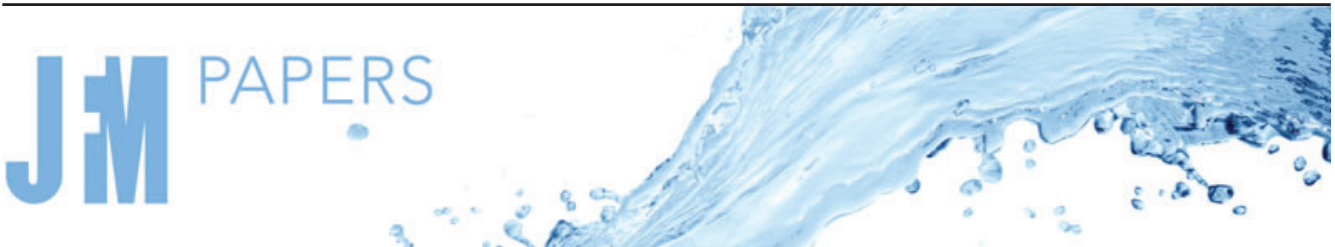

\section{Correlation-based flow decomposition and statistical analysis of the eddy forcing}

\author{
N. Agarwal ${ }^{1, \dagger}$, E.A. Ryzhov ${ }^{1,2}$, D. Kondrashov ${ }^{3,4}$ and P. Berloff ${ }^{1,5}$ \\ ${ }^{1}$ Department of Mathematics, Imperial College London, Huxley Building, London SW7 2AZ, UK \\ ${ }^{2}$ Pacific Oceanological Institute, Baltiyskaya 43, 690041 Vladivostok, Russia \\ ${ }^{3}$ Department of Atmospheric and Oceanic Sciences, University of California, Los Angeles, CA 90095, \\ USA \\ ${ }^{4}$ Institute of Applied Physics of the Russian Academy of Sciences, 603950 Nizhny Novgorod, Russia \\ ${ }^{5}$ Institute of Numerical Mathematics, Russian Academy of Sciences, Gubkina 8, 119333 Moscow, Russia
}

(Received 16 January 2021; revised 17 April 2021; accepted 29 June 2021)

We present a comprehensive study of the mesoscale eddy forcing in the ocean by proposing spatially local filtering of the high-resolution double-gyre ocean circulation solution into its large- and small-scale (eddy) components. The large-scale component is dominated by the mid-latitude gyres, the western boundary currents and their highly transient eastward jet extension; the eddy component is concentrated around the eastward jet and strongly interacts with it. The proposed decomposition method achieves flow filtering based on the local spatial correlations. This is different from the existing decomposition methods, e.g. classical Reynolds decomposition and moving-average (spatial) filtering with a constant filter size based on the first baroclinic Rossby deformation radius. Next, we characterize the dynamical impacts of the resulting eddy forcing on the large-scale flow in terms of their mutual time-lagged spatial correlations, formulated as product integral characteristics. Its temporal statistics uncover robust causality between the eddy forcing and the induced large-scale potential vorticity anomalies - referred to as the eddy backscatter. The results also prove the significance of the transient eddy forcing and the time lag dependence of the eddy backscatter. We argue that these properties are to be considered by eddy parametrization schemes. We further used the decomposed eddy fields to augment a coarse-resolution ocean model. The augmented solution statistically reproduces the missing eastward jet extension, enhances the eddy activities around it and recovers the essential large-scale low-frequency variability. This justifies a reduced-order

$\dagger$ Email address for correspondence: n.agarwal17@imperial.ac.uk

(C) The Author(s), 2021. Published by Cambridge University Press. This is an Open Access article, distributed under the terms of the Creative Commons Attribution licence (http://creativecommons.org/ licenses/by/4.0/), which permits unrestricted re-use, distribution, and reproduction in any medium, provided the original work is properly cited. 


\section{N. Agarwal, E.A. Ryzhov, D. Kondrashov and P. Berloff}

statistical emulation of the eddies - an emerging methodology for including eddy effects in non-eddy-resolving ocean models.

Key words: ocean circulation, quasi-geostrophic flows, ocean processes

\section{Introduction}

The ocean circulation is a complex, multiscale turbulent phenomenon characterized by many different space and time scales, and by coherent flow features, such as jets, vortices, gyres, etc., all nonlinearly interacting with each other. When the circulation is modelled, the underpinning nonlinearity manifests itself on a new level, each time the spatio-temporal resolution of the numerical model is refined (e.g. Shevchenko \& Berloff 2015). Therefore, all oceanic general circulation models (GCMs) include parametrizations of the unresolved subgrid-scale (eddy) effects on the large-scale motions, even at the (so-called) eddy-permitting resolutions. Numerous deterministic (Holloway 1987; Gent \& McWilliams 1990; Gent et al. 1995; Zanna et al. 2017; Berloff 2018) and stochastic (Frederiksen 1999; Berloff 2005; Crommelin \& Vanden-Eijnden 2008; Mana \& Zanna 2014; Gugole \& Franzke 2020) parametrization approaches for the oceanic mesoscale eddies demonstrate the spectrum of ideas and vigorous intensity of the ongoing research (reviewed by Hewitt et al. 2020). A new emerging trend is the development of stochastic eddy parametrizations which are data-driven rather than physics-based. Within this approach, the available observations can be used to train some underlying statistical model of the eddies or their essential properties via different methods (e.g. Kondrashov \& Berloff 2015; Kondrashov, Chekroun \& Berloff 2018; Bolton \& Zanna 2019; Ryzhov et al. 2020).

Among the many problems associated with the development of accurate and efficient eddy parametrizations, one problem is a reliable decomposition of a turbulent flow into resolved and unresolved (subgrid) scale components. Note that the 'resolved/unresolved' scale terminology is different from 'large-/eddy-scale' adjectives, as the former type mostly relates to the numerical modelling results (at different resolutions), whereas the latter to intrinsic scale separation using statistical decompositions. Nevertheless, in both cases, scale separation is problematic, because the ocean circulation possesses multiple and intricately interacting active scales without a spectral gap. In this study, we aim to focus on the problem of efficient scale separation, review the existing methods, propose a novel scale decomposition method and perform an extensive analysis of the resulting eddy effects.

The Reynolds flow decomposition (into the static time mean and fluctuations around it) is the classical choice in turbulence theory, but it does not provide any information on the spatio-temporal scales that characterize the decomposed flow fields. Furthermore, it only deals with the time-mean state and the corresponding statistical balances which is not acceptable from both the eddy parametrization perspective and pragmatic ocean modelling. Information on the temporal scales is not that important, since the time resolution is quite adequate in GCMs, but in the spatial domain the situation is different. This is because GCMs are limited by the horizontal grid resolution and, therefore, initially require parametrizations to deal with the horizontal length scales. Recently, moving-average decomposition methods with appropriate kernel sizes/shapes (Leonard 1975) have found applications in numerous ocean modelling problems (e.g. Zanna et al. 2017; Aluie, Hecht \& Vallis 2018; Berloff 2018) and in other types of turbulence (Piomelli et al. 1991; Vreman, Geurts \& Kuerten 1994; Aluie \& Eyink 2009). Such decompositions, unlike the Reynolds one, yield spatio-temporal variabilities in both 
large- and small-scale flow components and, therefore, allow to study their instantaneous nonlinear interactions. For mesoscale eddies it may sound reasonable to choose the kernel size based on the first baroclinic Rossby deformation radius, e.g. several radii. However, using a single characteristic length scale (even spatially varying) for spatially inhomogeneous and multiscale oceanic subgrid scale fields, developed on the underlying, inhomogeneous and multiscale resolved flow, is an obvious simplification that has to be verified against the actual length scales operating in the flow.

Researchers also use the spatial resolutions of the coarse- and fine-grid solutions to guide their filter size, but the issue of choosing the right one for determining the subgrid scales (for analyses and parametrization) is a lot more complicated than filtering the high-resolution solution down to the coarse resolution. This is because (i) there is no clear scale separation, and (ii) the nominally resolved flow features at the coarse grid may not necessarily be dynamically resolved, e.g. derivatives and nonlinearities may be misrepresented. Such model resolutions may be referred to as the 'grey zone' of partial resolution, and they arise due to the fact that a typically resolved dynamical feature, such as advection terms with strong nonlinear interactions, requires at least approximately 10 grid intervals to represent the underlying dynamics correctly. This implicit requirement can be even worse because of the non-local scale interactions. This typical situation complicates the choice of an appropriate moving-average kernel size/shape and suggests an exploration of the broad range of filters or even alternative dynamical filtering approaches (e.g. Berloff, Ryzhov \& Shevchenko 2021); this is precisely what motivates us in this work. We use an alternative purely statistical approach for achieving large/eddy scale separation with no explicit hyperparameter involved (such as the filter size in case of simple moving average). We emphasise that finding an objective way to separate eddies is a fundamental, critically important and unresolved problem. One of the goals of our study is to intensify its discussion for developing more accurate eddy parametrizations.

In the first part of this paper, we propose a statistically consistent correlation-based flow decomposition method that employs the Gaussian filtering kernel with geographically varying topology - consistent with the observed local spatial correlations - to achieve the scale separation. We term the decomposed flows as the large-/eddy-scale fields because we consider simulations from only one model resolution, and intend to decompose it into the flow components where one represents the solutions of a typical non-eddy-resolving ocean model and the other presents the missing eddying features. For the reference oceanic flow, we consider an eddy-resolving solution of the classical midlatitude double-gyre quasigeostrophic (QG) circulation.

The second part of our study aims to reveal dynamical interactions between the eddies and large-scale flow, such as eddy backscatter - characterized by the feedbacks of eddies on the large-scale flow via the transient part of the eddy forcing. These interactions are generally parametrized by a stochastic forcing in comprehensive ocean circulation models (Kraichnan 1976; Leith 1990; Frederiksen \& Davies 1997; Duan \& Nadiga 2007; Nadiga 2008; Kitsios, Frederiksen \& Zidikheri 2012; Grooms \& Majda 2013). Ryzhov et al. (2019) showed that improving a low-resolution model by additionally forcing it with the coarse-grained eddy forcing history inferred from a high-resolution model solution by standard flow decomposition is possible, and our study aims to elaborate on the involved mechanism and the roles of different filters. Here, we define the time-lagged product integral characteristic and use its time series to study the eddy-mean dynamical feedbacks, as given by the proposed flow decomposition and the other standard decompositions. Before doing this, we obtain the eddy forcings and study their statistical properties, which include spatio-temporal correlation scales to elucidate the differences between two statistically very different yet interacting flow fields. Finally, we verify the dynamical 
relevance of the correlation-based decomposition (CBD) eddy field by supplying it to augment a coarse-resolution model and by studying the augmented solution (similar to that done by Ryzhov et al. 2020). The augmented solution shows a significant reconstruction of the jet and its ambient eddy variability, as well as recovers the missing intrinsic large-scale low-frequency variability. In comparison, an equivalent fixed-size moving-average filter fails to achieve any of these improvements. This suggests that low-order data-driven modelling based on our treatment of the eddies is promising for use in coarse-resolution ocean circulation models.

The paper is organized as follows. Section 2 describes the double-gyre model and its eddy-resolving reference solution; $\S 3$ discusses the proposed flow decomposition method (i.e. part one of the two described above); $\S 4$ presents the eddy effects on the large-scale flows (i.e. the second part); and $\S 5$ discusses the results and conclusions.

\section{Double-gyre ocean circulation model and the reference solution}

The eddy-resolving reference solution is obtained from the double-gyre QG ocean circulation model operating at a large Reynolds number and representing an idealized North Atlantic (or North Pacific) circulation (Berloff 2015). The flow dynamics is governed by the following three-layer QG potential vorticity (PV) equations:

$$
\begin{gathered}
\frac{\partial q_{1}}{\partial t}+J\left(\psi_{1}, q_{1}\right)+\beta \frac{\partial \psi_{1}}{\partial x}=\frac{1}{\rho_{1} H_{1}} W(x, y)+v \nabla^{4} \psi_{1}, \\
\frac{\partial q_{2}}{\partial t}+J\left(\psi_{2}, q_{2}\right)+\beta \frac{\partial \psi_{2}}{\partial x}=v \nabla^{4} \psi_{2}, \\
\frac{\partial q_{3}}{\partial t}+J\left(\psi_{3}, q_{3}\right)+\beta \frac{\partial \psi_{3}}{\partial x}=-\gamma \nabla^{2} \psi_{3}+v \nabla^{4} \psi_{3},
\end{gathered}
$$

where $q_{i}$ and $\psi_{i}$ denote PV anomalies (PVAs) and velocity streamfunctions, respectively, for layers $i=1,2$ and 3. Here, $J($,$) denotes the Jacobian operator; \beta$ is the meridional gradient of the planetary vorticity; the isopycnal layer $i$ has density $\rho_{i}$ at the state-of-rest layer thickness $H_{i}$; the basin is a square with $2 L=3840 \mathrm{~km}$ side; and the only external forcing is given by the asymmetric stationary wind curl $W(x, y)$ :

$$
\begin{array}{ll}
W(x, y)=-\frac{\pi \tau_{0} A}{L} \sin \left[\frac{\pi(L+y)}{L+B x}\right], & y \leq B x, \\
W(x, y)=+\frac{\pi \tau_{0}}{L A} \sin \left[\frac{\pi(y-B x)}{L-B x}\right], & y>B x,
\end{array}
$$

where $A$ is the asymmetry parameter, $B$ is the non-zonal tilt parameter and $\tau_{0}$ is the wind-stress amplitude. Partial-slip boundary conditions are employed on the lateral walls, which involve first- and second-order derivatives normal to the boundary,

$$
\frac{\partial^{2} \psi_{i}}{\partial n^{2}}-\frac{1}{\alpha} \frac{\partial \psi_{i}}{\partial n}=0,
$$

with $\alpha=120 \mathrm{~km}$ as a boundary sublayer length scale. The asymmetrically forced double-gyre solutions becomes non-physical in the free- and no-slip limits (Berloff \& McWilliams 1999). The wind forcing is only in the top-layer (2.1a), whereas the eddy viscosity (with $v$ as the coefficient) acts in all layers $((2.1 a)-(2.1 c)$ ), and the bottom friction (with coefficient $\gamma$ ) acts in the deepest layer (2.1c). The PVAs $q_{i}$ from the 


$\begin{array}{ll}\text { Model Parameters } & \text { Values } \\ \text { Basin size (square) } & 3840 \mathrm{~km} \\ \text { Total depth } & 4 \mathrm{~km} \\ \text { Layer depths } & \mathrm{H}_{1}=250 \mathrm{~m}, \mathrm{H}_{2}=750 \mathrm{~m} \text { and } \mathrm{H}_{3}=3000 \mathrm{~m} \text {. } \\ \text { Spatial Resolution } & 7.5 \mathrm{~km} \\ \text { Time step } & 1 \text { day } \\ \tau_{0} & 0.08 \mathrm{~N} \mathrm{~m}^{-2} \\ \text { A } & 0.9 \\ \beta & 2 \times 10^{-11} \mathrm{~m}^{-1} \mathrm{~s}^{-1} \\ v & 10 \mathrm{~m}^{2} \mathrm{~s}^{-1} \\ \gamma & 4 \times 10^{-8} \mathrm{~s}^{-1} \\ f_{0} & 0.83 \times 10^{-4} \\ R d_{1} \text { and } R d_{2} & 40 \mathrm{~km} \text { and } 20.6 \mathrm{~km} \text {, respectively } \\ \text { Boundary Conditions } & \text { Partial slip } \\ \text { Partial-slip parameter }(\alpha) & 120 \mathrm{~km}\end{array}$

Table 1. Parameter values of the idealized ocean circulation model.

prognostic $((2.1 a)-(2.1 c))$ are inverted into the corresponding streamfunctions using the vorticity-streamfunction relationship given by the diagnostic equations:

$$
\begin{gathered}
q_{1}=\nabla^{2} \psi_{1}+S_{1}\left(\psi_{2}-\psi_{1}\right), \\
q_{2}=\nabla^{2} \psi_{2}+S_{21}\left(\psi_{1}-\psi_{2}\right)+S_{22}\left(\psi_{3}-\psi_{2}\right), \\
q_{3}=\nabla^{2} \psi_{3}+S_{3}\left(\psi_{2}-\psi_{3}\right) .
\end{gathered}
$$

The stratification parameters are given as

$$
S_{1}=\frac{f_{0}^{2}}{H_{1} g_{1}^{\prime}}, \quad S_{21}=\frac{f_{0}^{2}}{H_{2} g_{1}^{\prime}}, \quad S_{22}=\frac{f_{0}^{2}}{H_{2} g_{2}^{\prime}}, \quad S_{3}=\frac{f_{0}^{2}}{H_{3} g_{2}^{\prime}},
$$

where $g_{1}^{\prime}, g_{2}^{\prime}$ are reduced gravities corresponding to the two isopycnal interfaces and are chosen such that the baroclinic deformation radii, $R d_{1}, R d_{2}$, given as

$$
R d_{1}=g_{1}^{\prime} \frac{\sqrt{H_{1} H_{2}}}{f_{0} \sqrt{H_{1}+H_{2}}}, \quad R d_{2}=g_{2}^{\prime} \frac{\sqrt{H_{2} H_{3}}}{f_{0 \sqrt{H_{2}+H_{3}}}},
$$

are equal to $40 \mathrm{~km}$ and $20.6 \mathrm{~km}$, respectively. No-normal-flow is assumed on all boundaries for inversion, and the resulting Dirichlet boundary condition is determined using the mass conservation constraint at each layer. The model was discretized on a uniform grid with $513^{2}$ grid points and $7.5 \mathrm{~km}$ spatial resolution. The equations were solved with an efficient numerical scheme called CABARET (Karabasov, Berloff \& Goloviznin 2009). The reference solution was obtained for a total of 15000 days $(\approx 41$ years), after being spun up for approximately 100 years, and saved daily (in terms of PVA snapshots), which is adequate for representing the eddies. Parameters of the model and other details can be found in table 1 .

The flow snapshots (figure 1) clearly show two asymmetric gyres of opposite circulation, separated by a strong eastward jet, formed due to the merging western boundary currents and nonlinear eddy interactions. The flow is an idealization of the Gulf Stream in the North Atlantic and Kuroshio in the North Pacific. 

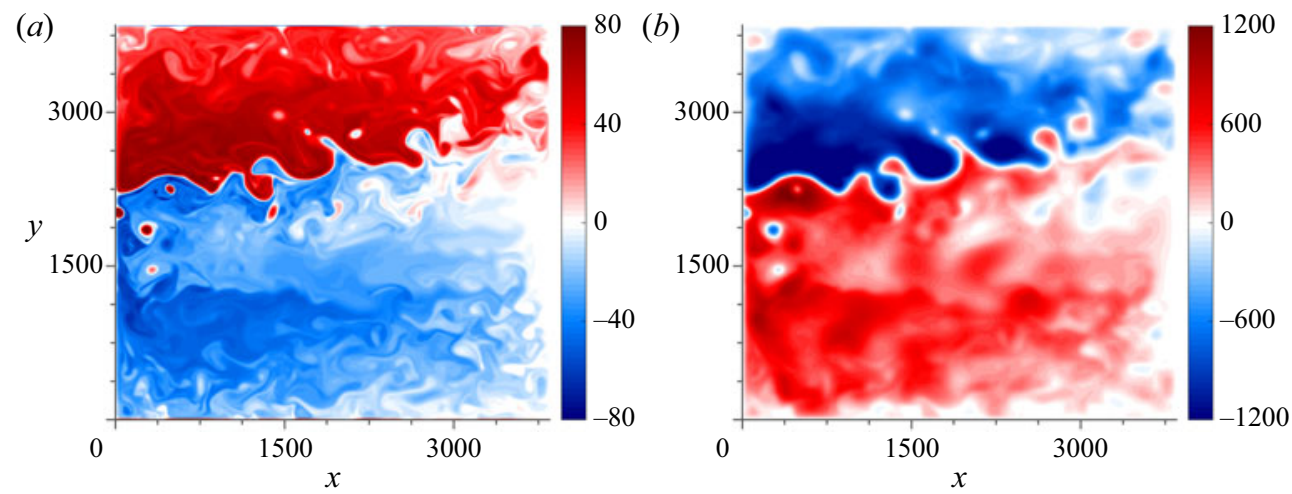

Figure 1. Snapshots of the reference solution. Shown are evolving upper-layer fields: $(a)$ PVA, and $(b)$ velocity streamfunction. The colourbars are in dimensionless units, with the length scale equal to the grid interval $7.5 \mathrm{~km}$ and velocity scale $0.01 \mathrm{~m} \mathrm{~s}^{-1}$. The positive (red) and negative (blue) values of PVA correspond to the cyclonic and anticyclonic motions, respectively.

For simplicity, from this point onward, we will show our results and analysis mostly for the top and middle layers, since the results for the bottom and middle layers are, usually, qualitatively similar (to be mentioned where they differ).

\section{Flow decomposition}

In this paper, our first goal is to implement a non-uniform, statistically constrained filtering of the reference flow fields into the large-scale and eddy components. Next, we want to compare the outcome with that of the moving-average filtering based on the baroclinic Rossby deformation radius, and to carry out a comprehensive analysis of the resulting eddy forcing and its relation to the large-scale flow. The generally decomposed streamfunction and PVA fields can be written as

$$
\psi(x, t)=\bar{\psi}(\boldsymbol{x}, t)+\psi^{\prime}(\boldsymbol{x}, t), \quad q(\boldsymbol{x}, t)=\bar{q}(\boldsymbol{x}, t)+q^{\prime}(\boldsymbol{x}, t),
$$

where the bars and primes indicate the large-scale and eddy fields, respectively.

Several different methods to obtain $(3.1 a, b)$ exist in the literature (e.g. Sagaut 2006), such as Reynolds decomposition, moving-average filtering, spectral filtering, etc. As discussed in $\S 1$, Reynolds decomposition provides no information about the separated scales in eddy-mean decomposed fields, and the latter two, generally, use a constant filter size for the entire domain - parts of which are evolving on entirely different length/time scales. For this reason, we propose an alternative flow decomposition with spatially-varying filter size across the domain, as discussed below. The application and results from moving-average and spectral filterings are provided in Appendices A and B, respectively.

\subsection{Correlation-based decomposition}

In this section, we discuss a non-uniform flow decomposition method developed here using local correlation information. This method augments the moving-average filtering while exploiting its advantages. The idea is to filter around each location according to the local length scale of the spatial correlation, as opposed to using a fixed kernel size based on the Rossby deformation radius (for QG this is equivalent to the same kernel size for the whole domain). Below the method is described in detail. 


\section{Correlation-based decomposition and eddy forcing analysis}

Let us consider the spatio-temporal flow field $\mathcal{F}$ to be decomposed. For any reference spatial location $x_{0}=\left(x_{0}, y_{0}\right)$, we first compute its zero-lag cross-correlation with every other spatial location in the domain (note that every spatial location in $\mathcal{F}$ has a time series) to obtain the corresponding spatial correlation map $C\left(\boldsymbol{x}_{0}, \boldsymbol{x}\right)$, and then fit a two-dimensional (2-D) Gaussian function to $\left|C\left(x_{0}, x\right)\right|$ - the absolute value of the correlation map - to diagnose the effective correlation length scale. The absolute value is used because we are only interested in finding locations with a strong influence on $x_{0}$; so the sign of the correlation does not matter.

Figure $2(a, d, g, b, e, h)$ shows correlation maps $C\left(\boldsymbol{x}_{0}, \boldsymbol{x}\right)$ for reference locations $\boldsymbol{x}_{0}$ in the eastward jet, subpolar gyre and subtropical gyre regions, all in the upper-ocean PVA field (i.e. $\mathcal{F}=q_{1}$ ). The correlation maps (figure $2 a, d, g$ ) show that the correlations are spatially local in both the jet and the gyre regions and decay exponentially away from the reference locations. A zoomed-in view of the correlations around the reference locations (figure $2 b, e, h$ ) further demonstrates that the correlations are nearly isotropic in the subpolar gyre and eastward jet regions but are significantly anisotropic in the subtropical gyre region. For a few reference locations in the subtropical gyre region, we also found the local correlations to be anisotropic at a certain angle (not shown here), whereas those along the western boundary were found to be aligned with the meridional direction, due to the strong western boundary currents. Therefore, to precisely account for the correlation geometry, we fitted a rotated anisotropic Gaussian function positioned at $x_{0}$ to the full correlation map $\left|C\left(x_{0}, x\right)\right|$ and determined the effective length scale using the standard deviation of the Gaussian along each axis.

The form of the Gaussian function used is

$$
f\left(x, y ; x_{0}, y_{0}\right)=\exp \left(-X^{2} / a^{2}\right) \exp \left(-Y^{2} / b^{2}\right),
$$

where $X$ and $Y$ are the rotated and translated coordinate axes, given as

$$
\left[\begin{array}{l}
X \\
Y
\end{array}\right]=\left[\begin{array}{cc}
\cos \theta & \sin \theta \\
-\sin \theta & \cos \theta
\end{array}\right]\left[\begin{array}{c}
x-x_{0} \\
y-y_{0},
\end{array}\right]
$$

and $a$ and $b$ are the standard deviations along them, respectively. Note, that at the reference location $\boldsymbol{x}=\boldsymbol{x}_{0}$, both $f$ and $C$ are equal to 1 . The fitted Gaussians (figure $2 c, f, i$ ) appropriately assign the weights according to the decay of the correlations away from the reference location and adjusts the shape according to the direction and extent of anisotropy. The values of $a$ and $b$ determine the correlation length scale operating along each direction, whereas the overall effective correlation length scale (say, $\mathcal{L}$ ) can be illustrated by $\mathcal{L}=\sqrt{a b}$. Fitting such a Gaussian function $f$ to the correlation map for each $\boldsymbol{x}_{0}$ produces the spatial maps $a(\boldsymbol{x} ; \mathcal{F}), b(\boldsymbol{x} ; \mathcal{F}), \theta(\boldsymbol{x} ; \mathcal{F})$ and $\mathcal{L}(\boldsymbol{x} ; \mathcal{F})$ for the given flow field $\mathcal{F}$. The length scale maps $\mathcal{L}(x ; \mathcal{F})$ and the inferred anisotropy, computed as $\mathcal{A}(\boldsymbol{x} ; \mathcal{F})=a(x ; \mathcal{F}) / b(\boldsymbol{x} ; \mathcal{F})$ (gridpoint-wise division), for the three layers of PVA (i.e. $\left.\mathcal{F}=q_{i}, i=1,2,3\right)$ are shown in figure 3 . We do not show $\theta\left(\boldsymbol{x} ; q_{i}\right)$ as it is relatively noisy, and geographical variations in $\theta$ are not particularly interesting.

Significant geographical variations in $\mathcal{L}\left(\boldsymbol{x} ; q_{i}\right)$ attest multiscale and inhomogeneous properties of the mesoscale turbulence (figure $3 a, c, e$ ). In the upper ocean (figure $3 a$ ), the eastward jet region exhibits mean $\mathcal{L}\left(q_{1}\right)$ equal to $91 \mathrm{~km}$, which is comparable to the filtering based on $2.25 R d_{1}$, whereas the subpolar and subtropical gyres have mean correlation length scales equal to 108 and $133 \mathrm{~km}$, respectively. The mean and median length scale for the entire domain is roughly $105 \mathrm{~km}$, i.e. slightly higher than $2.5 R d_{1}$. A region of even larger $\mathcal{L}(\boldsymbol{x})$ exists in the centre of the basin in the middle layer (figure $3 c$ ), where typical $\mathcal{L}$ values are $250-500 \mathrm{~km}$. This is because this part of the basin is dominated 
(a)



(d)

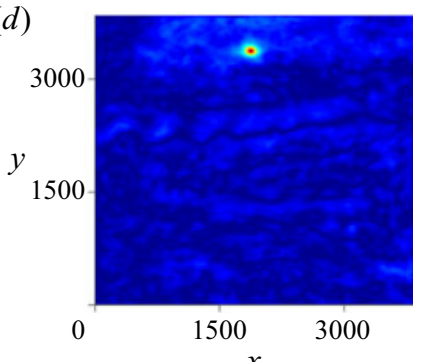

$(g)$

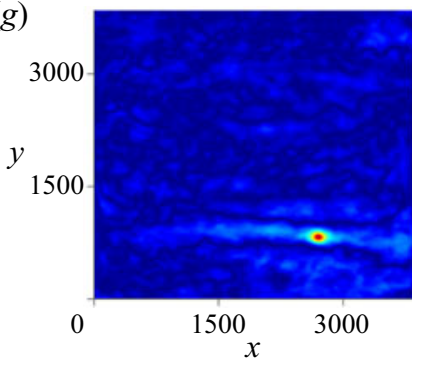

(b)

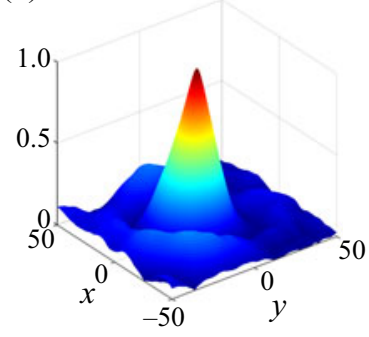

(e)



$(h)$

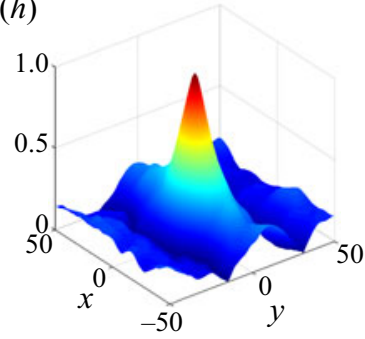

(c)

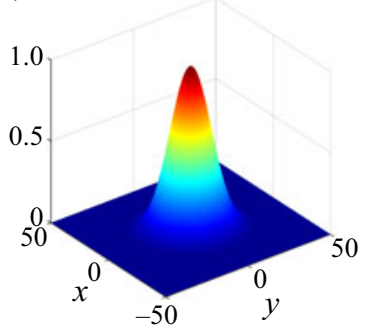

$(f)$

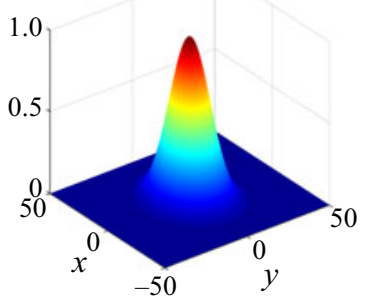

(i)

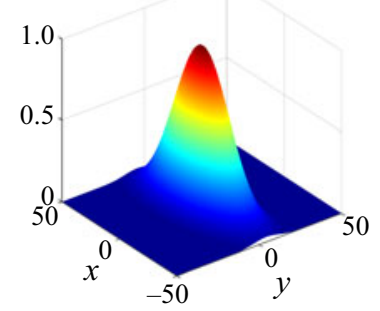

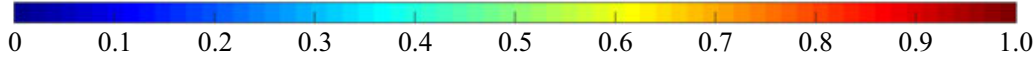

Figure 2. Visualisation of $\left|C\left(q_{1}\left(x_{0}, \boldsymbol{x}\right)\right)\right|$ contours $(a, d, g)$, its zoomed-in three-dimensional view around the reference location $x_{0}(b, e, h)$ and the fitted Gaussian function $(c, f, i)$ for three randomly chosen reference locations in the eastward jet $(a-c)$, the subpolar gyre $(d-f)$ and the subtropical gyre $(g-i)$ regions. The entire length of PV anomaly, equal to 15000 days, is used for computing cross-correlations. The $z$-axis in $(b, e, h)$ and $(c, f, i)$ represents the correlation magnitude and the Gaussian weights, respectively. These have been additionally colour coded to match the contour levels in $(a, d, g)$.

by a large-scale interdecadal variability pattern with long-range correlations (figure 4). Large differences between the correlation length scales at the two layers infer the need to emulate eddies as horizontally and vertically inhomogeneous, and multiscale entities. In the bottom layer (figure $3 e), \mathcal{L}(x)$ is relatively short along the western boundary and long along the eastern one (south of the eastward jet). This is consistent with the prevalence of small-scale eddies in the former region and long waves radiating from the eastern boundary in the latter one.

The $\mathcal{A}\left(x ; q_{i}\right)$ maps (figure $\left.3 b, d, f\right)$ infer that the correlations are mostly anisotropic in the large-length-scale regions, except near the western and eastern boundaries, where it is inherently stretched in the meridional direction (i.e. $a / b<1$ ). Anisotropy along the lateral boundaries is due to the strong western boundary current and zonal radiation from the eastern boundary (it is more intense in deep layers). The $\mathcal{A}(\boldsymbol{x})$ values for the top-layer $\mathrm{PV}$ anomaly (figure $3 b$ ) also infer that the correlations in the jet region are nearly isotropic 

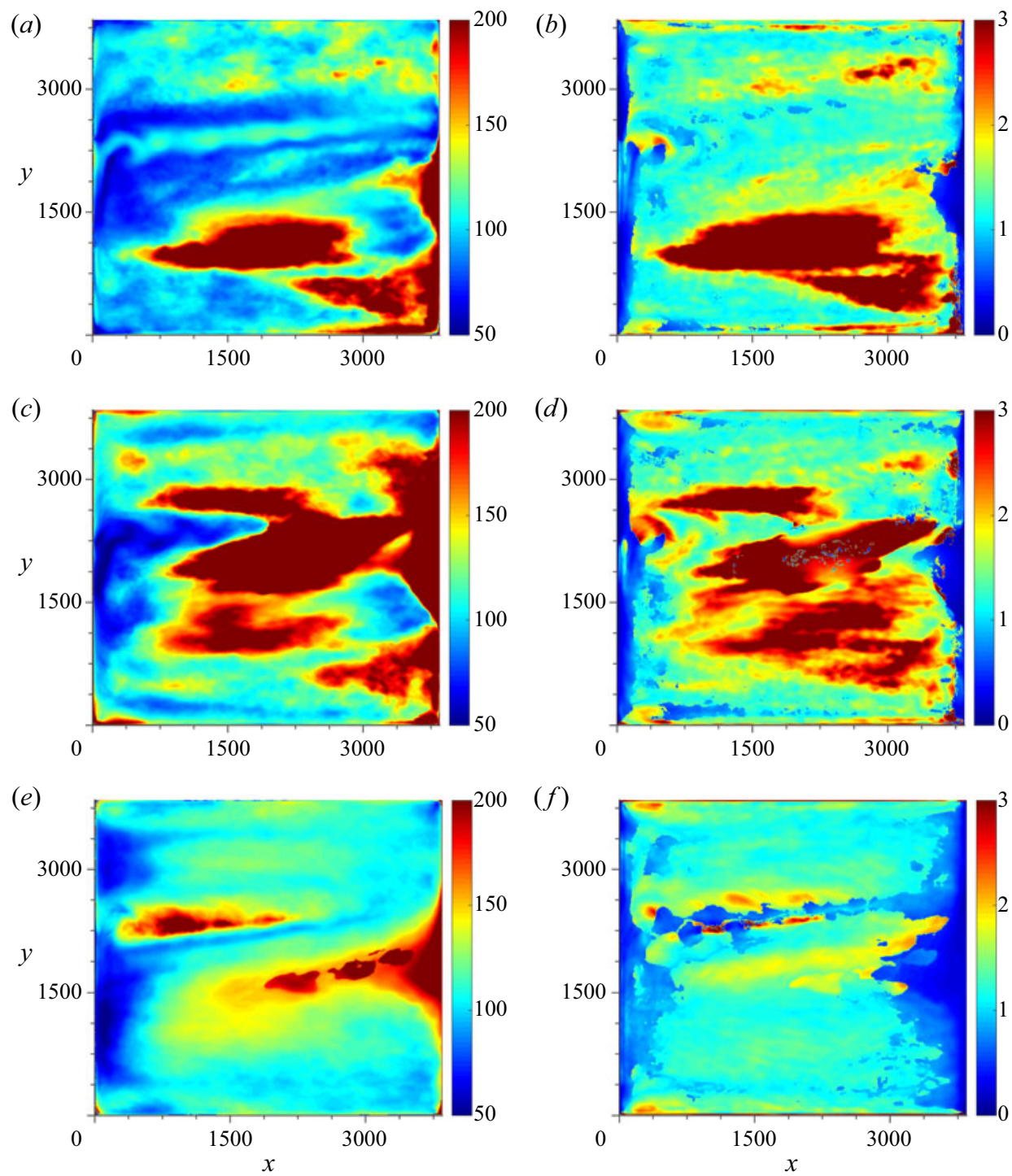

Figure 3. Maps of correlation length scale $\mathcal{L}(\boldsymbol{x} ; q)(a, c, e)$ and the correlation anisotropy $\mathcal{A}(\boldsymbol{x})=a / b(b, d, f)$ for the three layers of PV anomaly in the top $(a, b)$, middle $(c, d)$ and bottom $(e, f)$ layers. The units of length scale are in $\mathrm{km}$, and the anisotropy estimates are dimensionless.

- mainly due to the rich eddy activities in this region leading to localised coherent isotropic structures. However, in the deeper layers (figure $3 d, f$ ), as the eastward jet gets weaker, the flow around it becomes more anisotropically correlated with its neighbouring locations.

The length scale and anisotropy maps together reveal the complex and multiscale nature of the flow, and suggest that a fixed-size kernel can substantially underfilter or overfilter eddies depending on the location; therefore, a differential filter size over the domain is justified. For practical applications, correlation length scales are to be determined from observational data and, in some cases, from eddy-resolving model solutions. Note that the CBD provides additional flexibility of imbuing temporal dependency in the correlation length scales by employing time-lagged correlation (i.e. $C\left(x_{0}, \boldsymbol{x} ; \tau\right)$, where $\tau$ is the 


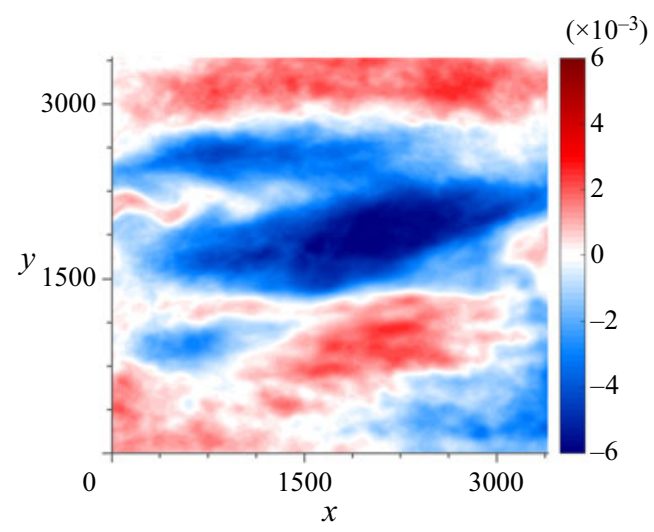

Figure 4. The empirical orthogonal function (EOF) representing the large-scale interdecadal variability in $q_{2}$, responsible for producing large $\mathcal{L}$ in the middle of the domain (see figure $3 c$ ). The decorrelation time scale for this pattern is roughly 16.7 years, as obtained from the temporal autocorrelation of its principal component. A stripe of 30 grid points is removed from all four boundaries of $q_{2}$ before its EOF decomposition to get this as the first EOF; otherwise, boundary trapped eddies dominate a large chunk of leading EOFs, thus making it harder to detect this variability. Units are non-dimensional.

anticipated time scale of variation in the length scales) instead of instantaneous correlation $\left(C\left(x_{0}, x\right)\right)$ discussed here.

We filtered each spatial location of the PVA fields using a rotated anisotropic Gaussian kernel as defined in (3.2), with the diagnosed parameters $a, b$ and $\theta$. This can be expressed as

$$
\bar{q}\left(x_{i}, y_{j}, t\right)=\sum_{i^{\prime}} \sum_{j^{\prime}} G\left(x_{i^{\prime}}, y_{j^{\prime}} ; x_{i}, y_{j}\right) q\left(x_{i^{\prime}}, y_{j^{\prime}}, t\right) ; \quad 1 \leq i^{\prime}, j^{\prime} \leq 513
$$

where $G$ is the normalized Gaussian kernel,

$$
G\left(x_{i^{\prime}}, y_{j^{\prime}} ; x_{i}, y_{j}\right)=\frac{f\left(x_{i^{\prime}}, y_{j^{\prime}} ; x_{i}, y_{j}\right)}{A_{f}}
$$

and $A_{f}=\sum_{i^{\prime}} \sum_{j^{\prime}} f\left(x_{i^{\prime}}, y_{j^{\prime}} ; x_{i}, y_{j}\right) \equiv \pi a b$ is the sum of the Gaussian weights per (3.2). Note that only the grid points within the Gaussian ellipse would contribute significantly in (3.4) because the Gaussian weights decay exponentially with distance from the ellipse centre.

Using (3.4) and (3.1a,b), the large- and small-scale components of PVA fields were obtained for each layer, and the corresponding streamfunctions were obtained by inversion of (2.4) (figure 5). On comparing these eddy fields with the corresponding full flow snapshots (figure 1), we see (especially for $\psi_{1}$ ) vigorous eddy activity along the eastward jet extension and near boundaries. Note that the process can be reversed, i.e. by filtering streamfunction fields first, then by obtaining PVA using (2.4); however, we found that this algorithm generates discontinuities in PVA upon the differentiation (note that the inversion is intrinsically a smoother operation). 

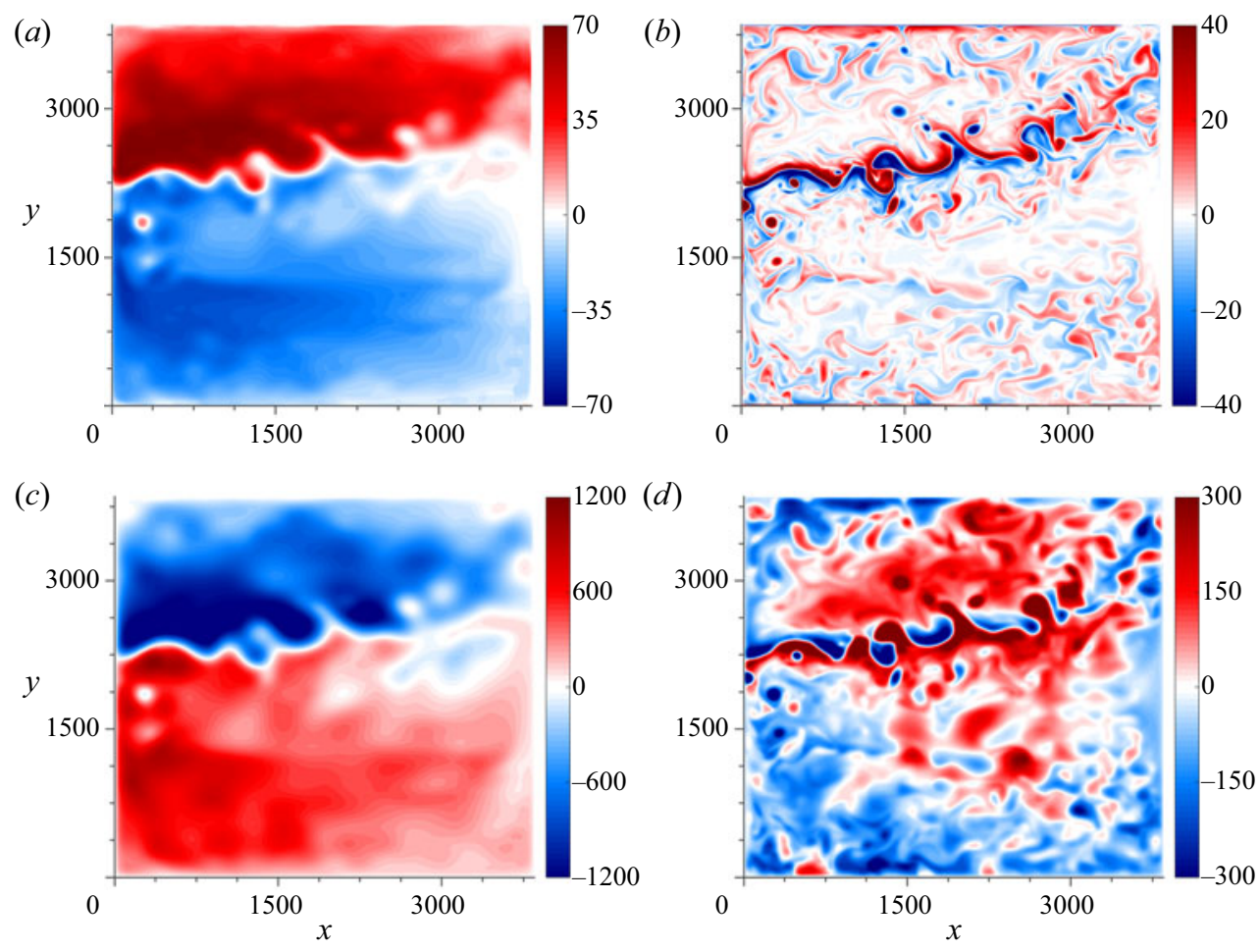

Figure 5. Snapshots of $(a) \bar{q}_{1},(b) q_{1}^{\prime}$, (c) $\bar{\psi}_{1},(d) \psi_{1}^{\prime}$ obtained using the CBD method. $(a, b)$ show that gyres and the eastward jet are well captured by the large-scale flow component; $(c, d)$ shows vigorous eddies all over the basin but with the largest concentration in the eastward jet region and around the boundaries. The colour scales are in non-dimensional units.

\section{Results}

\subsection{CBD eddy forcing}

In the following, we analyse eddy feedbacks on the large-scale flow by considering eddy forcing, which is argued to play an important role in the maintenance of large-scale flow features such as the eastward jet and its adjacent recirculation zones (Nadiga 2008; Shevchenko \& Berloff 2016). Revealing the essential and poorly understood statistical characteristics of the eddy forcing is our next goal. This information should help to improve statistical eddy models by constraining them to preserve these characteristics.

The eddy forcing is obtained by substituting the decomposed flow $(3.1 a, b)$ into the governing equation (2.1), and by rewriting it as the evolution equation for the large-scale component as (illustrated only for the top layer but applicable for each layer)

$$
\frac{\partial\left(\bar{q}_{1}+q_{1}^{\prime}\right)}{\partial t}+J\left(\bar{\psi}_{1}+\psi_{1}^{\prime}, \bar{q}_{1}+q_{1}^{\prime}\right)+\beta \frac{\partial\left(\bar{\psi}_{1}+\psi_{1}^{\prime}\right)}{\partial x}=\frac{1}{\rho_{1} H_{1}} W(x, y)+v \nabla^{4}\left(\bar{\psi}_{1}+\psi_{1}^{\prime}\right),
$$

which on rearrangement gives

$$
\frac{\partial \bar{q}_{1}}{\partial t}+J\left(\bar{\psi}_{1}, \bar{q}_{1}\right)+\beta \frac{\partial \bar{\psi}_{1}}{\partial x}=\mathcal{H}\left(\bar{q}_{1}, \bar{\psi}_{1}\right)+\mathcal{I}\left(q_{1}^{\prime}, \psi_{1}^{\prime}\right)+\mathcal{N}\left(\bar{q}_{1}, \bar{\psi}_{1}, q_{1}^{\prime}, \psi_{1}^{\prime}\right)
$$


where

$$
\begin{gathered}
\mathcal{H}\left(\bar{q}_{1}, \bar{\psi}_{1}\right)=\frac{1}{\rho_{1} H_{1}} W(x, y)+v \nabla^{4} \bar{\psi}_{1}, \\
\mathcal{I}\left(q_{1}^{\prime}, \psi_{1}^{\prime}\right)=-\frac{\partial q_{1}^{\prime}}{\partial t}-\beta \frac{\partial \psi_{1}^{\prime}}{\partial x} \\
\mathcal{N}\left(\bar{q}_{1}, \bar{\psi}_{1}, q_{1}^{\prime}, \psi_{1}^{\prime}\right)=-J\left(\psi_{1}^{\prime}, \bar{q}_{1}\right)-J\left(\bar{\psi}_{1}, q_{1}^{\prime}\right)-J\left(\psi_{1}^{\prime}, q_{1}^{\prime}\right) .
\end{gathered}
$$

Clearly, the final expression (4.2) provides both linear $\mathcal{I}\left(q_{1}^{\prime}, \psi_{1}^{\prime}\right)$ and nonlinear $\mathcal{N}\left(\bar{q}_{1}, \bar{\psi}_{1}, q_{1}^{\prime}, \psi_{1}^{\prime}\right)$ terms expressing the feedbacks from the eddy- to large-scale fields. The linear term was found to be much smaller than the nonlinear one (also seen by Ryzhov et al. 2019). Therefore, we approximated the eddy forcing by the nonlinear term $\mathcal{N}$, i.e.:

$$
\begin{aligned}
E_{i}(x, y ; t) & =-\left[J\left(\bar{\psi}_{i}, q_{i}^{\prime}\right)+J\left(\psi_{i}^{\prime}, \bar{q}_{i}\right)+J\left(\psi_{i}^{\prime}, q_{i}^{\prime}\right)\right] \\
& =-\left[J\left(\psi_{i}, q_{i}\right)-J\left(\bar{\psi}_{i}, \bar{q}_{i}\right)\right],
\end{aligned}
$$

where $i$ is the layer index, overbar indicates the large-scale component and prime indicates the eddy component; the others are the full fields. The eddy forcing can also be written in the flux divergence form as

$$
E_{i}(x, y ; t)=-\left[\nabla \cdot u_{i} q_{i}-\nabla \cdot \bar{u}_{i} \bar{q}_{i}\right],
$$

where $\boldsymbol{u}$ is the non-divergent geostrophic velocity. Note that this is different from the commonly used large-eddy simulation (LES)-based definition of instantaneous eddy forcing:

$$
E_{i}^{L E S}(x, y ; t)=-\left[\nabla \cdot \overline{u_{i} q_{i}}-\nabla \cdot \overline{\boldsymbol{u}}_{i} \bar{q}_{i}\right] .
$$

The differences between the expressions are as follows. First, (4.6) is only applicable to the filtering methods which commute with partial derivatives (otherwise $\nabla \cdot \overline{\boldsymbol{u}_{i} q_{i}}$ becomes $\left.\overline{\nabla \cdot \boldsymbol{u}_{i} q_{i}}\right)$. So, it does not hold for the CBD, as it involves a spatially varying filtering kernel and does not commute with spatial derivatives. However, (4.5) is more flexible and holds for all filtering methods, as it is obtained by direct substitution of the filtered fields into the governing equation. Second, for $E^{L E S}$, we need the same characteristic length scales of $\boldsymbol{u} q, \boldsymbol{u}$ and $q$, but they are unlikely to be the same. Because we cannot filter the governing equations using multiple filters, the whole process is either infeasible or leads to inconsistent results if an approximate length scale is used for all three. In contrast, $E$ is easier to generalize for filtering based on variable kernel sizes and shapes. Moreover, it only involves the filtering of $\boldsymbol{u}$ and $q$, which are connected by the PV inversion. Third, the dynamics of $E$ has clearly interpreted nonlinear advection terms, whereas the nonlinear terms in $E^{L E S}$ are harder to interpret (e.g. they are not advection) because they involve double filterings.

We diagnosed $E$ from the data using the energy and enstrophy conserving Arakawa's Jacobian discretization (Arakawa 1966). From the spatio-temporal statistics of $E$, it is clear that the eddy forcing is most intense in the upper-ocean eastward jet region, and it weakens away from it and becomes more homogeneously distributed with depth (figure $6 a, b$ ). The time-mean $E_{1}$ (figure $6 c$ ) has very small values in the jet region and even less elsewhere. By decomposing $E_{1}$ into its time-mean $\left(\left\langle E_{1}\right\rangle(x, y)\right)$ and transient $\left(E_{1}^{\prime}(x, y, t)\right)$ components, we found that in the eastward jet region the standard deviation of $E_{1}^{\prime}$ is an order of magnitude larger than $\left\langle E_{1}\right\rangle$ (figure $6 d$ ), thus, suggesting that $E_{1}^{\prime}(x, y, t)$ dominates over $\left\langle E_{1}\right\rangle(x, y)$. 

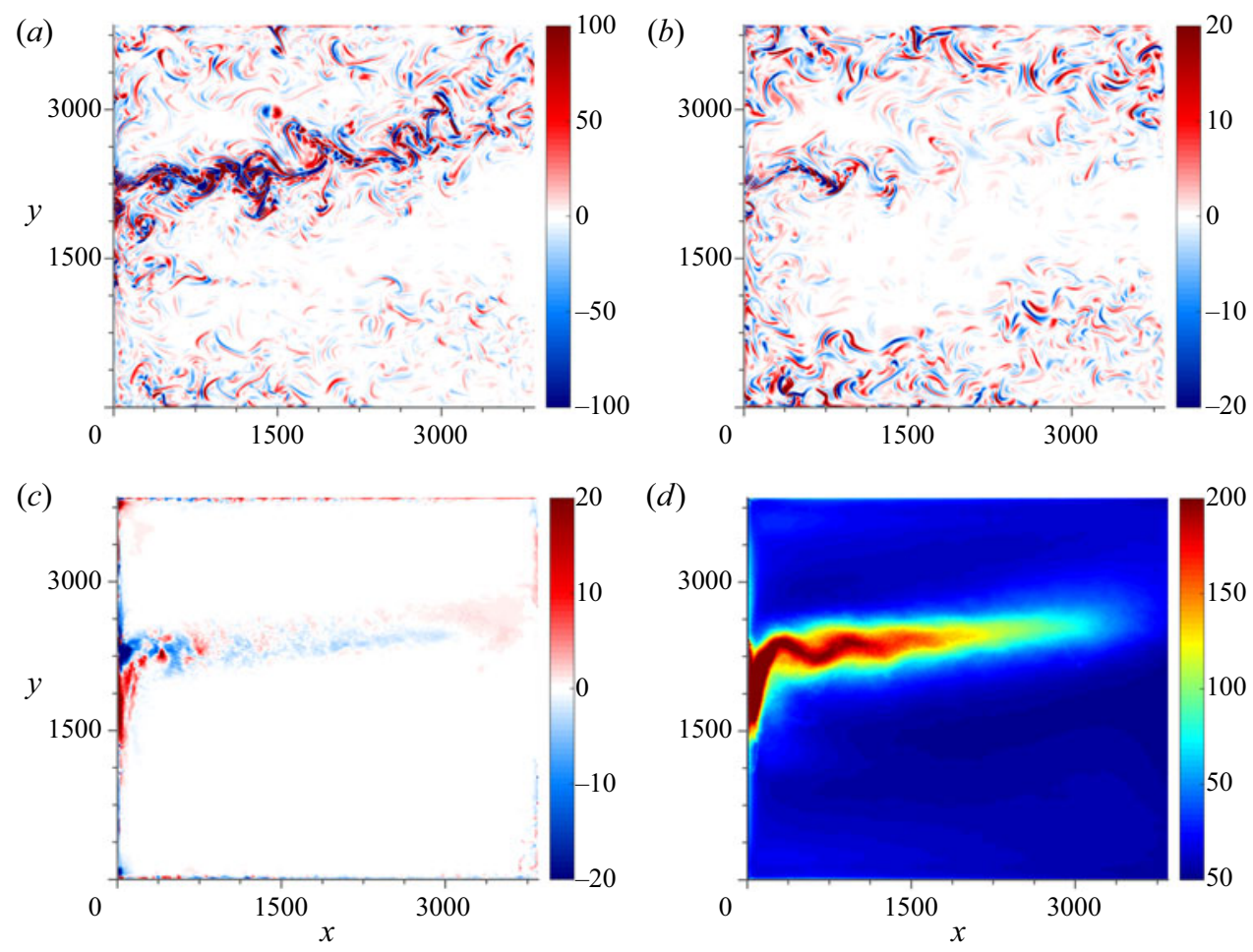

Figure 6. Patterns of the instantaneous eddy forcing $E$ from CBD for the $(a)$ top and the $(b)$ middle isopycnal layers. The colourbars are in non-dimensional units. Clearly, the eddy forcing is concentrated in the upper-ocean eastward jet region. $(c)$ Time-mean and $(d)$ standard deviation of $E_{1}$. The time-mean field is weaker by an order of magnitude, suggesting that the eddy forcing feedback is mostly due to the fluctuations, which are most pronounced in the jet region.

To characterize the correlation length scales of the eddy forcing, we used the Gaussian fitting method described in $\S 3.1$ and obtained the length scale map $\mathcal{L}\left(x ; E_{i}\right)$ and the anisotropy map $\mathcal{A}\left(\boldsymbol{x} ; E_{i}\right)$. The $\mathcal{L}\left(\boldsymbol{x} ; E_{1}\right)$ map (figure $7 a$ ) shows approximately $20 \mathrm{~km}$ long correlation scales around the eastward jet and in the subpolar gyre region, but they are approximately $5 \mathrm{~km}$ longer to the south of the jet and towards the eastern boundary. The latter region of relatively long correlation length scales extends out with depth (figure $7 b$ ), but the eastward jet region still retains short $\mathcal{L}$ values. This shows that the eddy forcing is the most tightly correlated where it is the most intense. Furthermore, the ratio of the $\mathcal{L}$ values for the PVA and eddy forcing fields shows that, on average, the eddy forcing $\mathcal{L}$ values are approximately 5 times smaller (figure $3 a, c, e$ ) than the PVA ones, both globally and around the eastward jet. Since one needs at least two grid intervals to approximate a length scale, this implies that at least 10 grid intervals are needed to represent an eddy forcing dynamically. The eddy forcing $E$ is expected to be dominated by small spatial scales as all the computations are performed on the fine grid and that it is a triply differentiated field (in streamfunction) involving the eddy fields. Small-scale dominance of the eddy forcing is also true for the standard LES-type eddy forcing, e.g. the one derived by Mana \& Zanna (2014) using a similar QG ocean model. Furthermore, the $\mathcal{A}\left(\boldsymbol{x} ; E_{1}\right)$ map (figure $7 c$ ) implies that the eddy forcing correlations are nearly isotropic in the entire domain (mean $\mathcal{A}(\boldsymbol{x})=1.1$ ), but relatively more so in the subpolar gyre (mean $\mathcal{A}(\boldsymbol{x})=1$ ) than in the eastward jet and the subtropical gyre region (mean $\mathcal{A}(\boldsymbol{x})=1.2$ ). 
(a)



(c)

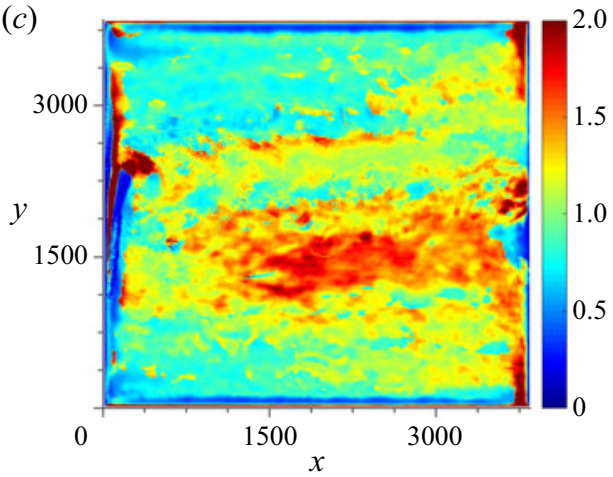

(b)

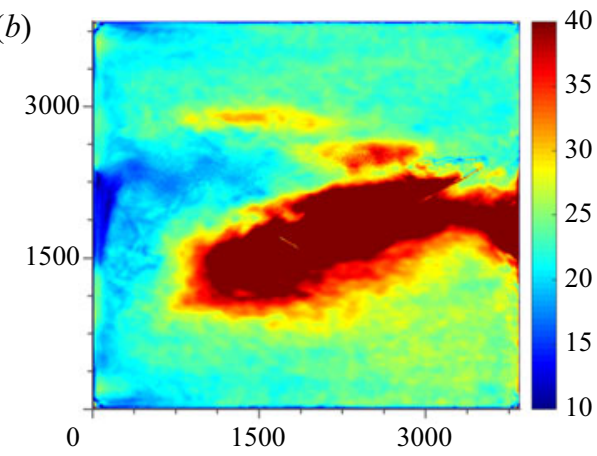

(d)

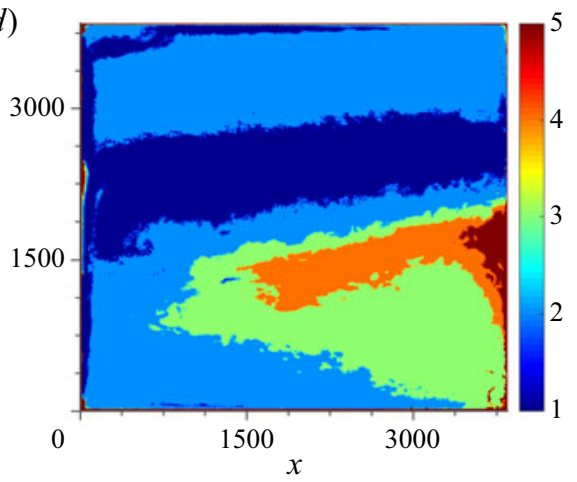

Figure 7. Correlation length scale maps $\mathcal{L}(\boldsymbol{x})$ (in km) for the CBD-decomposed outputs: (a) $E_{1}$ and (b) $E_{2}$, (c) the anisotropy map $\mathcal{A}\left(x ; E_{1}\right)$ and $(d)$ the time scale map (in days) for $E_{1}$. Relative to PVAs, the eddy forcing is characterized by significantly smaller length and time scales (compare with figure 3(a,c,e); the time scale map for PVA is not shown for brevity).

To characterize the eddy forcing further, we diagnosed the temporal scale for each grid point - defined as the lag at which the corresponding autocorrelation drops by a factor of exp from the zero-lag value - and analysed the resulting correlation time scale map. The fast evolving nature of the eddy forcing is visible in its top-layer time scale plot (figure $7 d$ ). In particular, the eastward jet region is dominated by the shortest correlation time scales and is characterized by the average correlation time scale of 1 day, which is approximately 3 times shorter than in the interior gyres and is approximately 25 times shorter than PVA time correlations in the eastward jet region (not shown for brevity). Locally, eddy forcing on the north and south flanks of the eastward jet has correlation time scales of approximately 1.8 and 3.5 days, respectively, whereas its basin-averaged value is approximately 2.7 days. Overall, the spatio-temporal correlation study of the eddy forcing suggest that it can be modelled as a stochastic field with short spatio-temporal correlations in the regions where it is most intense.

In comparison, $q_{1}$ yields correlation time scales of 25 days in the eastward jet and $\sim 10^{4}$ days in the gyre regions (not shown). The corresponding basin averaged correlation time is $~ 9500$ days, which is more than 3500 times that for the eddy forcing - annotating the differences between the two very different yet interacting flow fields.

Therefore, we learned that (i) the eddy forcing is characterized by grid-scale level correlation in space and $O(1)$ days correlation in the temporal domain - much smaller than the induced PVA field, (ii) the eddy forcing is least spatially correlated where it is the most intense - the jet eastward region, and (iii) the transient component of the eddy 

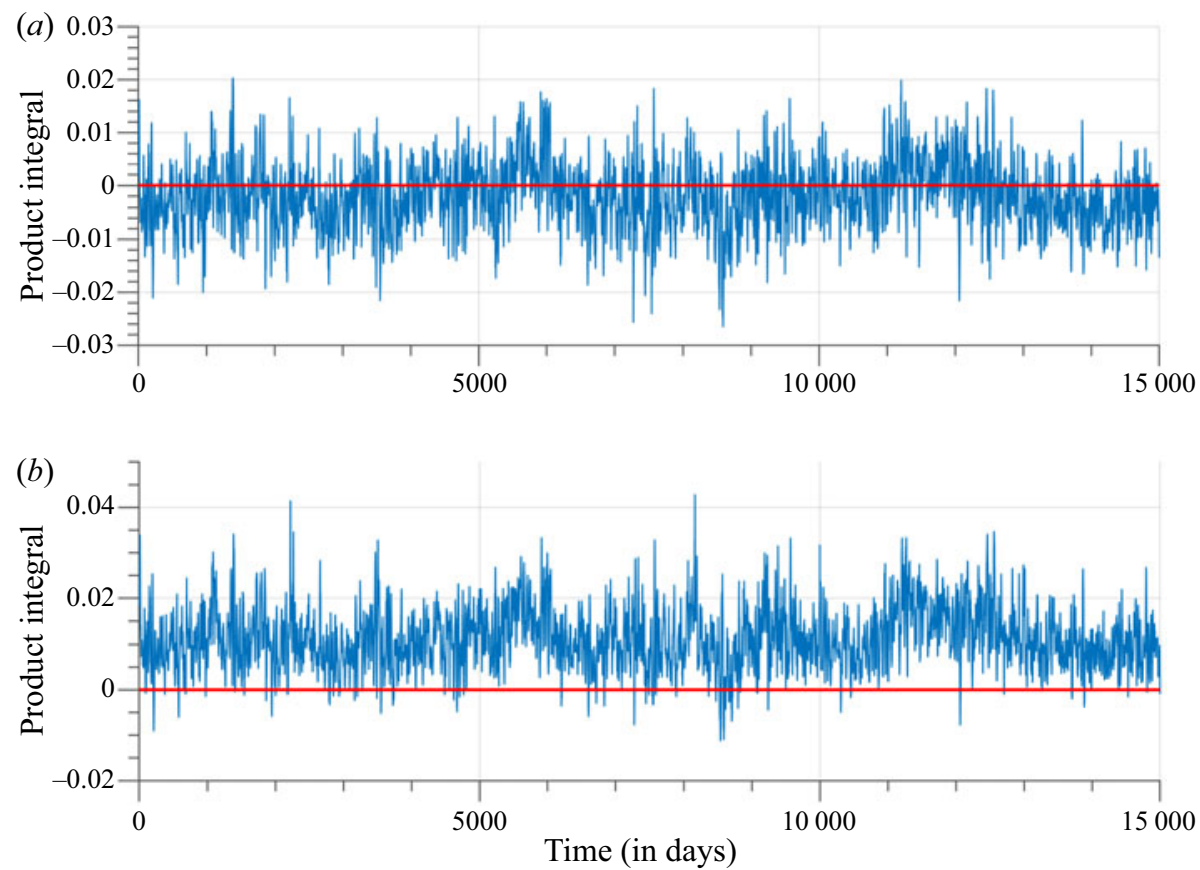

Figure 8. Product integral $I(t)$ between $\bar{q}_{1}$ and coarse-grained $E_{1}$ for $(a) \tau=0$ and $(b) \tau=1$ day. The red lines indicate $I(t)=0$, which is used to define instants of positive $I$. The curve in $(b)$ illustrates presence of the underlying eddy backscatter, as it shows positive correlation between the eddy forcing and the large-scale flow response for most of the time.

forcing is more dominant than its time-mean. How exactly can this small-scale and highly transient eddy forcing feed back on the much larger and slower evolving large-scale flow features? Studying this process is the main subject of the next section.

\subsection{Product integral analysis}

Next, we intend to study the dynamical characteristics of the eddy forcing and the induced large-scale flow, and quantify their possible interactions using a suitable metric. The goal here is to illuminate the eddy backscatter mechanism, which supports the eastward jet and its adjacent recirculation zones, and, thus, is characterized statistically by a positive correlation between the transient eddy forcing and large-scale flow response. To achieve this, we used an approach based on analysing the product integral $I(t)$, defined instantaneously as the spatial integral of the point-wise (Hadamard) product $\odot$ of two standardized snapshots (i.e. point-wise subtracting the time-mean and dividing by the standard deviation) from flow fields, say, $\mathcal{F}_{1}$ and $\mathcal{F}_{2}$ :

$$
I(t)=\frac{1}{A} \iint_{\Omega} \hat{\mathcal{F}}_{1}(x, y ; t) \odot \hat{\mathcal{F}}_{2}(x, y ; t) \mathrm{d} x \mathrm{~d} y,
$$

where $A$ is area of the domain $\Omega$.

We calculated $I(t)$ for $\mathcal{F}_{1}=\bar{q}_{1}$ and $\mathcal{F}_{2}=E_{1}$. The resulting instantaneous product integral was found to have mixed positive and negative values with a negative time-mean (figure $8 a$ ), but this value should be treated cautiously, as the two flow variables (i.e. $\bar{q}_{1}$ and $E_{1}$ ) evolve on very different space and time scales (as shown in $\S \S 4.1$ and 3.1), and the 

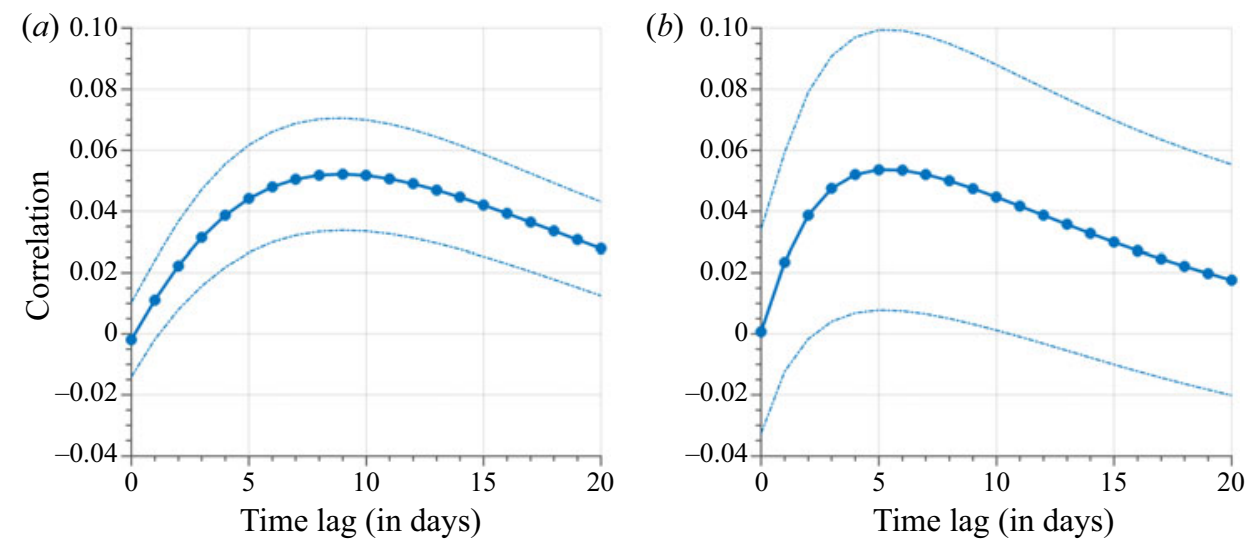

Figure 9. Plot of the time-lag dependence of the product integral correlation between coarse-grained $E_{1}$ and $\bar{q}_{1}(a)$ for the full domain and $(b)$ for the eastward jet region. The jet region was defined over the locations where the standard deviation of $E_{1}$ is more than 50 (in non-dimensional units). The two dashed lines indicate $I(t)$ values within two standard deviations from the mean. Both $(a, b)$ characterize the eddy backscatter and its response time scale.

flow response to eddy forcing may accumulate over time rather than being instantaneous. Therefore, a time-lagged extension of (4.7) was applied to get more insight into the relation between the eddy forcing and the large-scale flow response after time lag $\tau$, i.e.

$$
I(t, \tau)=\frac{1}{A} \iint_{\Omega} \hat{\mathcal{F}}_{1}(x, y ; t+\tau) \odot \hat{\mathcal{F}}_{2}(x, y ; t) \mathrm{d} x \mathrm{~d} y,
$$

with $\mathcal{F}_{1}=\bar{q}_{1}$ and $\mathcal{F}_{2}=E_{1}$. The time-lagged $I(t, \tau)$ curve with $\tau=1$ day (figure $8 b$ ) clearly shows a positive feedback from the eddy forcing to the flow response, and, therefore, suggests the eddy backscatter mechanism. Such a positive time-lagged correlation between $E_{1}$ and $\bar{q}_{1}$ is not obvious, because (i) there are other terms in the governing equation, and (ii) no such lagged correlation exists for white-noise forcing in the same governing equation. By considering the time-lagged relation, we advanced the results of Berloff (2018) where only zero lag was considered, and the resulting product integral (between large-scale PV anomaly and eddy forcing) was found to be positive due to the excessively large filter size - equal to $5 R d_{1}$. Therefore, the correct conclusion was based on the less general diagnostics. Here, our time-lagged detection of the main eddy backscatter characteristics is a lot more solid and substantially reinforces the previous conclusions. Dynamical interpretation of this result is that the flow adjustment due to the eddy feedback happens not instantly but with some delay. To see if the eastward jet region is particularly susceptible to the eddy backscatter, we repeated the analyses only for the eastward jet region, but the outcome was the same.

We considered values of $\tau$ up to 20 days, to find that the time-mean product integral $\langle I(t, \tau)\rangle=(1 / T) \int_{0}^{T} I(t, \tau) \mathrm{d} t$ - or spatial time-lagged correlation - increases with $\tau$ monotonically, peaks at around 9 days and then decays monotonically towards zero (figure $9 a$ ). The standard deviation of $\langle I(t, \tau)\rangle$ is also very small for small lags but increases thereafter, reaching the maximum at $\tau=9$ days. Note that although the values of $\langle I(t, \tau)\rangle$ are small, on randomizing $\widehat{E_{1}}$ in (4.8) we obtained three orders of magnitude smaller values, which inferred the significance of the illuminated spatio-temporal correlation between the two dynamically complex and structurally very different flow fields that were shown to evolve on different space and time scales. We refer to the time 
lag of the maximum correlation as the backscatter response time scale. Using a few other normalization techniques, such as $L_{2}$ norm, scaling between $[0,1]$, etc., we also proved the insensitivity of the $\tau$-dependence curve to the chosen normalization.

We also analysed the $\langle I(t, \tau)\rangle$ curve for the eastward jet region (figure $9 b$ ) and there are a few key differences to note: (i) the zero-time-lag correlation is small but positive in the jet region; (ii) the backscatter response time scale is almost half for the jet region, which proves the insignificance of long memory effects of the eddy feedback over there; (iii) for each $\tau$ the standard deviation of $I(t, \tau)$ is higher for the jet region than on average, thus indicating more multiscale nonlinear interactions.

Additionally, we explored the time-lagged product integral correlation between $\mathcal{F}_{1}=$ $\mathrm{d} \bar{q}_{1} / \mathrm{d} t$ and $\mathcal{F}_{2}=E_{1}$ and obtained positive instantaneous correlation between the two, which is also the maximum; and the correlation decreased monotonically with the lag. The magnitude of the correlation was also found to be somewhat higher in the eastward jet region. Together with the product integrals between $E_{1}$ and $\bar{q}_{1}$, it suggests that the positive (negative) part of the transient eddy forcing instantaneously drives the tendency of positive (negative) flow anomalies, and this effect manifests itself in the induced flow field after a definite time scale - different for the jet and the gyre regions. Note that this conclusion cannot be drawn only using the positive correlation seen between $E_{1}$ and $\partial \bar{q}_{1} / \mathrm{d} t$, as feedback between opposite polarities of $E_{1}$ and $\bar{q}_{1}$ anomalies is also possible under such a positive correlation.

Next, we studied the global and jet-regional $\langle I(t, \tau)\rangle$ curves for $\bar{q}_{1}$ and $E_{1}$ obtained using the fixed-size moving-average decomposition method with three filter sizes: 11, 20 and 40 grid intervals (hereafter referred to as F11, F20 and F40), which correspond to $82.5 \mathrm{~km}$, $150 \mathrm{~km}$ and $300 \mathrm{~km}$, respectively (in terms of $R d_{1}$, these are approximately $2 R d_{1}, 3.75 R d_{1}$ and $7.5 R d_{1}$ ). Note, that F11 is the most obvious choice for a filter size based on $R d_{1}$ when using the fixed-size moving average method; F20 and F40 are among other reasonable choices of larger filter sizes. Additionally, the mean length scale diagnosed for the top-layer PVA using CBD (figure $3 a$ ) is approximately equal to $105 \mathrm{~km}$ and, therefore, is in between the F11 and F20 filter sizes. For the full field correlation plot (figure 10a), the overall shape of the curve was the same for all cases, but F11 and CBD outcomes resembled each other the most; especially up to the backscatter response time scale, beyond which F11 correlations decayed faster. Overall, the CBD produced equal or higher correlations than F11, at all time lags. However, the outcomes were very different for the eastward jet region (figure $10 b$ ), as the CBD-produced eddy forcing showed a much higher correlation (with $\bar{q}_{1}$ ) than F11, and this correlation was strongest around the backscatter time scale, which was roughly equal to 5 days. In effect, the CBD-produced eddy forcing yields a stronger feedback on to the large-scale flow field in the eastward jet region, where the backscatter is most pronounced. On the other hand, F20 and F40 filtering kernels result in larger backscatter response time scales, both globally and in the eastward jet region. In general, the time scale increases with an increase in moving-average filter size, whereas the amplitude of correlation goes down. The full-domain zero-lag correlation is also small and positive for F20 and F40 filters, contrary to CBD and F11. A similar $\langle I(t, \tau)\rangle$ analysis for the interior gyres showed small differences between the F11 and CBD results, whereas the F20 and F40 ones differed vastly from them. For the subtropical gyre, F11 and CBD correlation curves resembled those for the full field, but, in the subpolar gyre, the F11 correlations remained generally lower than the CBD ones.

Overall, this suggests that the performance of moving average filters is critically sensitive to the choice of filter size, and looking for and developing a less subjective filtering approach is totally justified. We also tried including a spectral filtering approach 

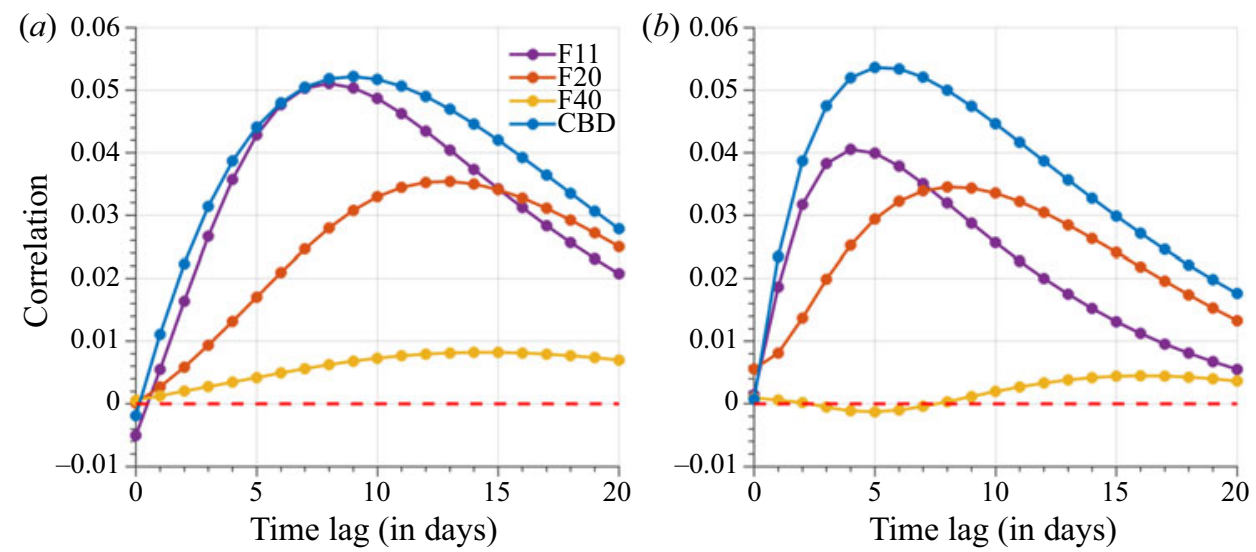

Figure 10. Comparison of the correlation curves (between coarse-grained $E_{1}$ and $\bar{q}_{1}$ ) from CBD and the three moving-average cases (F11, F20, F40) for $(a)$ the full domain and $(b)$ the eastward jet region. As the moving-average filter size increases, the correlation curves becomes flatter, their correlation amplitudes decrease, and the backscatter time scale information is lost. Also, CBD captures the highest correlation in the jet region, which indicates the backscatter in a most pronounced way.

in the comparison list but obtained highly noisy and inconsistent decomposed fields for $q_{1}$ - due to the non-locality of coherent vortices and the eastward jet region in the spectral domain (discussed and shown in Appendices B).

Therefore, considering the lack of knowledge about the optimal filter sizes for eddy-/large-scale decompositions, CBD provides a powerful alternative with no explicit hyperparameter dependence of its characteristics. Another advantage of CBD is its usefulness for the unstructured grid systems, where uniform moving average filtering can cause significant distortions. In the next section, we investigate the dynamical relevance of CBD eddy fields by using them to augment a low-resolution non-eddy-resolving model solution. In the future, we will exploit the comprehensive study done here to build low-order data-driven models for CBD eddy fields and use them for such low-resolution model augmentations.

\subsection{Augmentation of the coarse-resolution solution}

In this section, we test the dynamical relevance of the CBD-produced eddy fields by applying an augmentation procedure of the corresponding coarse-resolution model and compare the augmented solution against the high-resolution reference. The augmentation procedure is comprehensively introduced by Ryzhov et al. (2020). It involves supplying small-scale fields into the coarse-resolution model during its run, thus correcting its solution interactively as (valid for all three layers)

$$
\frac{\partial q_{c}}{\partial t}+J\left(\psi_{c}, q_{c}\right)+\beta \frac{\partial \psi_{c}}{\partial x}=\mathcal{H}\left(\psi_{c}, q_{c}\right)+\mathcal{E}\left(\psi_{c}, q_{c}, \psi^{\prime}, q^{\prime}\right)
$$

where the subscript $c$ denotes coarse-resolution (or low-resolution) solutions; $\mathcal{H}$ represents all terms involving the coarse-resolution solutions $\psi_{c}, q_{c}$; and $\mathcal{E}$ presents the interactive eddy forcing computed using the coarse-resolution solutions and the supplied eddy fields $\psi^{\prime}, q^{\prime}$ from the eddy-/large-scale decomposition of the high-resolution solution (the eddy fields are coarse grained to the available coarse resolution before adding). Note that the expression (4.9) is analogous to (4.2), but the coarse-resolution solutions $q_{c}$ and $\psi_{c}$ 
are not the same as $\bar{q}$ and $\bar{\psi}$ - this is a known problem in model augmentation and parametrization in general and addressing it is outside the scope of the paper. A similar coarse-resolution model augmentation technique can also be applied using the eddy forcing history $E$ (4.4a) itself (Ryzhov et al. 2019). However, the corresponding off-line eddy forcing history additionally involves the high-resolution ('true') large-scale fields, which is more demanding in terms of external data availability as compared with using only the high-resolution eddy field in the on-line eddy forcing computation. Moreover, our approach allows for the interactive (hence part of the solution) feedback from the eddy component to the large-scale one.

We considered an eddy permitting coarse-resolution model with a grid resolution of $129 \times 129$, compared with an eddy-resolving $513 \times 513$ for the high-resolution model. The coarse-resolution model by itself fails to produce the eastward jet (which is mostly eddy-driven) and its adjacent recirculation zones - attested both by the flow snapshot (figure 11a) and the temporal standard deviation (figure 11b); conversely, the high-resolution solution produces a pronounced eastward jet separating the two gyres (figure $11 c, d$ ).

For coarse-resolution augmentation, we (i) decomposed the high-resolution PVA into its large- and small-scale components (as described in $\$ 3.1$ ), followed by their inversions to get the corresponding streamfunctions, (ii) coarse-grained the eddy streamfunction to a $129 \times 129$ grid resolution by applying local averaging over 4 by 4 high-resolution grid intervals (the difference between the high- and coarse-resolution counterparts), (iii) obtained coarse-grained eddy PVA using the vorticity-streamfunction relationship (2.4), and finally (iv) added both eddy fields to the coarse-resolution model, as described in (4.9), through on-line calculation of eddy forcing at each time step (note that the coarse-grid $\psi^{\prime}$ and $q^{\prime}$ can also be obtained by coarse-graining $q^{\prime}$ directly in step (ii) and inverting it to get $\psi^{\prime}$ at the same resolution.). We spun up the coarse-resolution model - until it reached its statistically equilibrated state - before integrating it for an additional 15000 days of the available external small-scale fields, and comparing its statistical properties with those of the coarse- and high-resolution solutions (of similar time length). The augmentation is performed for both F11- and CBD-produced eddy fields, as the two filters provide nearly similar product integral correlations, as seen in the previous section. For both, the components of the eddy forcing are the corresponding coarse-resolution solutions and the externally supplied small-scale (eddy) fields.

The augmented flow snapshot for F11 (figure 11e) shows poor jet reconstruction and weak eddy activities around it, whereas the CBD augmented solution (figure $11 \mathrm{~g}$ ) shows considerably more eddying features and a stronger eastward and also meandering jet, with all these features being closer to the reference 'truth' - the high-resolution solution. The standard deviation of the augmented solutions for the two methods (figure $11 f, h$ ) further distinguishes between the induced variabilities around the jet region. The CBD produces much more variability around the jet, with more pronounced eastward extension of it, as compared with F11, which yields marginal improvement relative to the coarse-resolution model solution (figure 11b). However, there are still some notable differences between the standard deviation of the CBD-augmented solution and the high-resolution reference flow, as the latter shows even higher values near the eastward jet. This suggests that the evolving states of the CBD augmented model lie on a different attractor than the high-resolution reference (similar to that observed by Nadiga \& Livescu 2007). Consequently, this means that the supplied eddy fields operate differently than the corresponding perfect eddy field - i.e. those extracted from a perfect decomposition with $q_{c}=\bar{q}$ and the same initial condition for both high-resolution and the augmented model. Here, the eddy fields get quickly decorrelated from the coarse-grid model solution they are augmenting. 
N. Agarwal, E.A. Ryzhov, D. Kondrashov and P. Berloff
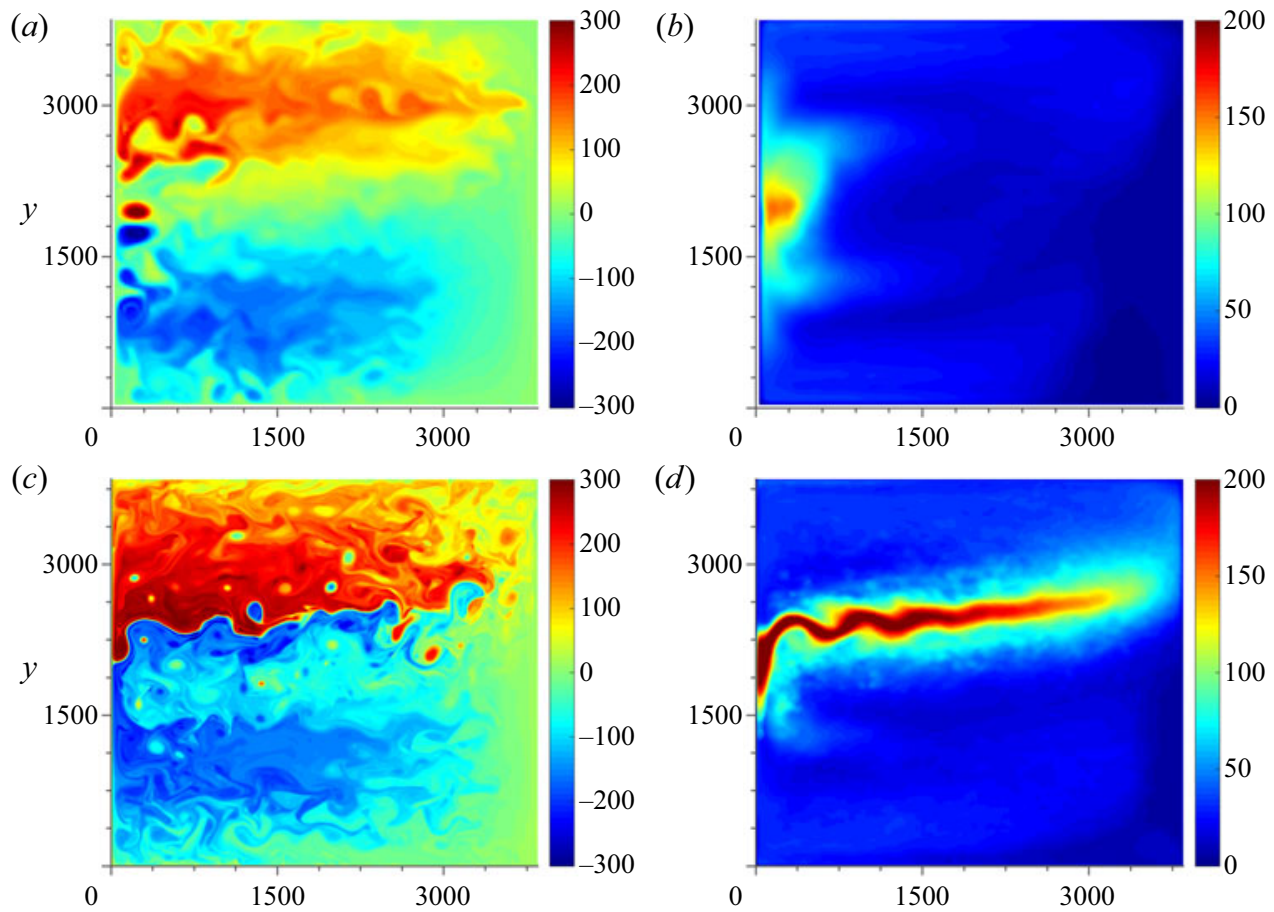

(e)
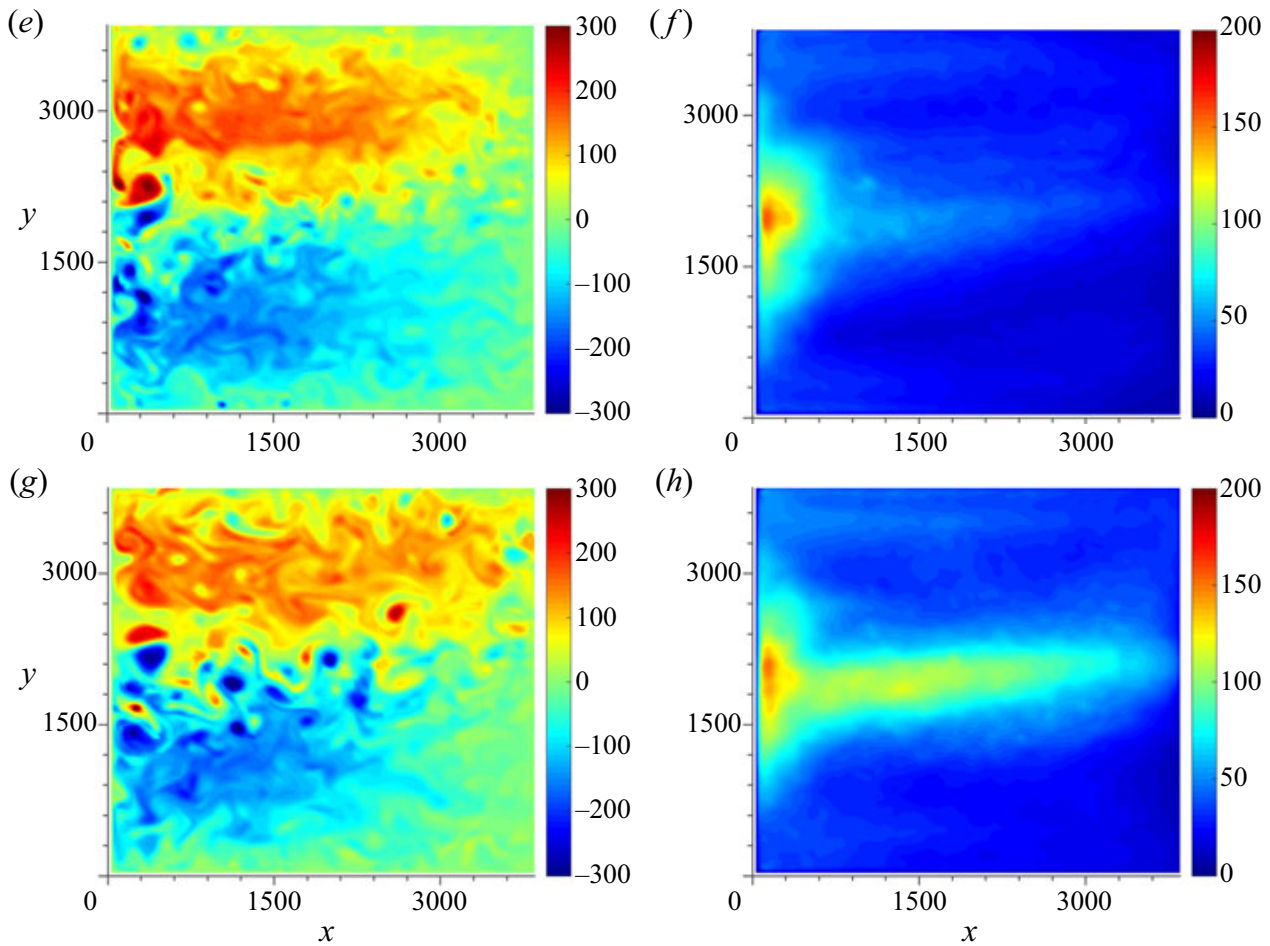

Figure 11. Comparison of the coarse-resolution, high-resolution, F11- and CBD-augmented model solution snapshots (panels $a, c, e, g$ ) of the top-layer PV anomaly and their standard deviations (panels $b, d, f, h$, respectively) for 15000 days of data. Starting from the top, the four rows belong to coarse-, high-resolution, F11- and CBD-augmented model solutions, respectively. 
Correlation-based decomposition and eddy forcing analysis
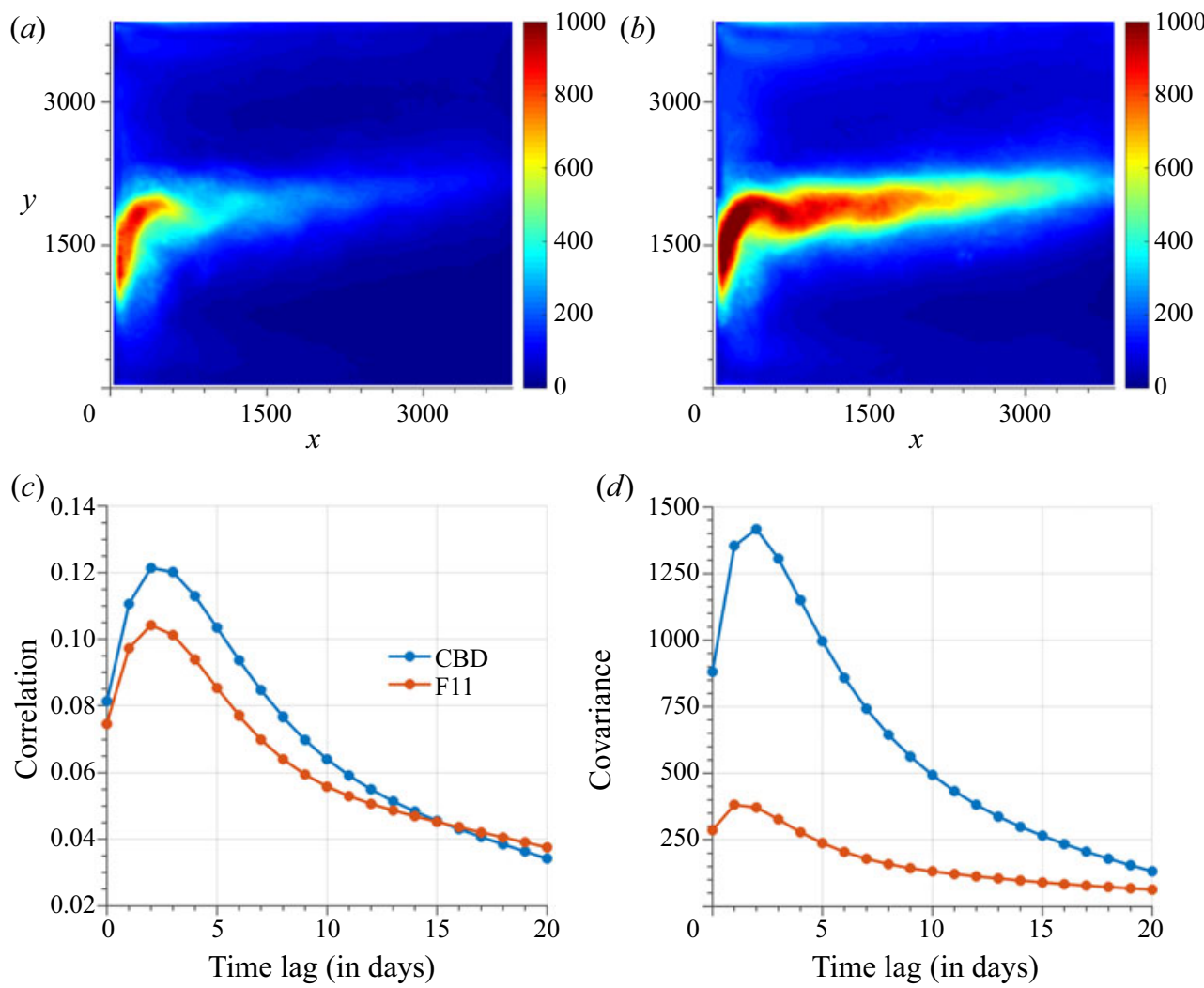

Figure 12. Comparison of the standard deviations of the online eddy forcing computed using (a) F11 and (b) CBD eddy fields for the coarse-resolution solution augmentation. (c) The product integral correlations between the augmented PV anomaly and the corresponding eddy forcings for F11 and CBD decompositions. (d) Same as (c) but covariance. The covariance is obtained by using (4.8) but without normalizing the two flow fields by their standard deviations.

This inherent property of the eddy fields makes them suitable for stochastic treatment to develop parametrizations while also accounting for the effects of initial condition perturbations.

The reason behind poor augmentation by the F11 eddy field is the weak interactive eddy forcing generated in conjunction with $\psi_{c}$ and $q_{c}$ (figure 12a). In comparison, the CBD eddy forcing (figure $12 b$ ) is much stronger and more eastward-extended than its F11 counterpart, thereby providing a much better augmentation. To understand the impact of this discrepancy in the eddy forcing on eddy backscatter, we analysed the corresponding product integral correlations between the augmented PVA and the online eddy forcing $\mathcal{E}$ (figure 12c). The results suggest that both F11 and CBD eddy forcings possess similar correlations with the induced PV anomaly, and the same backscatter time scale; the CBD eddy forcing is only marginally highly correlated with its induced PVA at short time-lags (1-10 days). Recall that, even in $\S 4.2$ (figure $10 a$ ) we found the product integral correlation (i.e. backscatter) characteristics of F11 and CBD to be nearly the same; the current results verify them for online eddy forcing. However, on analysing the corresponding covariances - obtained using (4.8) but without normalizing the two flow fields by their standard deviations - we found an order of magnitude of difference between F11 and CBD (figure $12 d$ ). The correlation and covariance results together suggest that, although the F11 


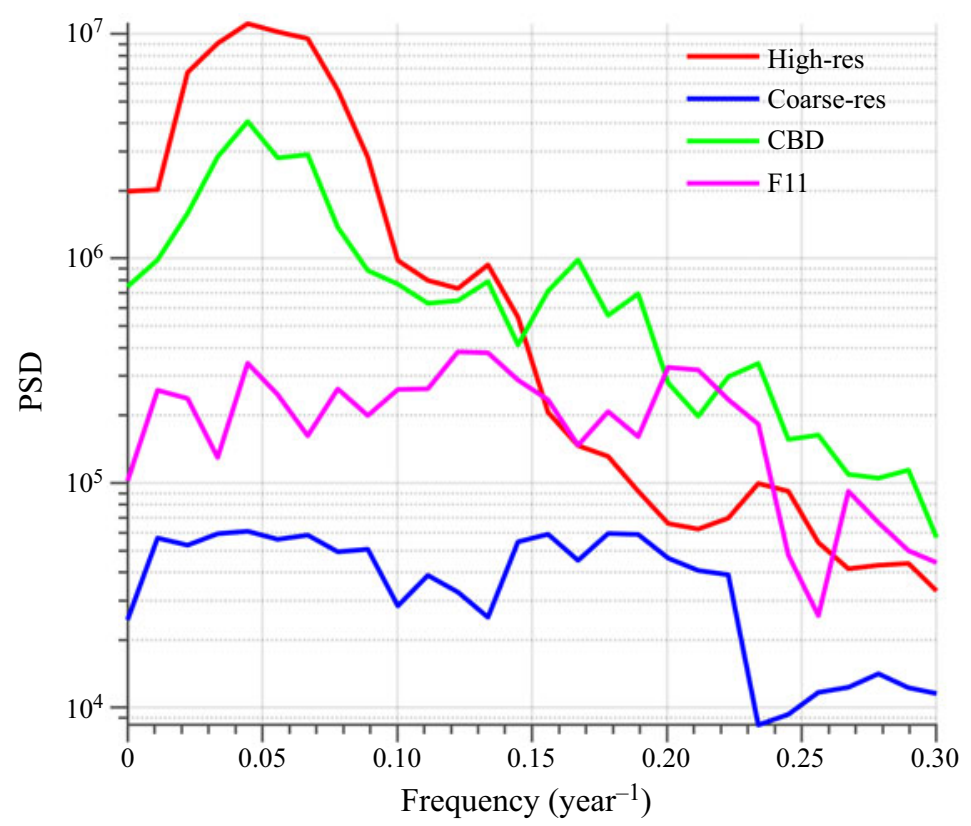

Figure 13. Comparison of the power spectral density (PSD) estimates for coarse-resolution, high-resolution, F11- and CBD-augmented solutions. The PSD is computed using the robust multitaper method, as introduced by Percival et al. (1993).

eddies feedback similarly on the induced PV anomalies, the low magnitude of its eddy forcing fails to deliver a significant jet reconstruction and, therefore, the resulting induced PVA is also weak compared with CBD, which shows significantly strong variabilities around the jet. This collective intensity of the eddy forcing and the induced dynamical response is captured in the covariance magnitude, which is an order of magnitude smaller for F11 than CBD. The contribution of the eddy forcing intensity alone on the covariance magnitude can be quantified using semi-covariance - i.e. those obtained using (4.8) with normalized induced PVA but un-normalized eddy forcing. Such a semi-covariance magnitude is also significantly high for CBD compared with F11 (not shown). It may sound sensible to use a larger filter size instead of F11 to enhance the eddy forcing strength and thereby improve the jet characteristics, but it is already shown in $\S 4.1$ that such big filter sizes (e.g. F20, F40) lead to poor backscatter; therefore, this is not an option.

Finally, we performed Fourier spectral analysis of the basin-average potential energy time series, given as

$$
P(t)=\frac{1}{2} \sum_{i=2}^{3} \frac{H_{i}\left(\delta_{i 2} S_{i 2}+\delta_{i 3} S_{i}\right)}{V} \iint_{\Omega}\left(\psi_{i}-\psi_{i-1}\right)^{2} \mathrm{~d} y \mathrm{~d} x,
$$

where $S_{i j}$ and $S_{i}$ are the stratification coefficients, $H_{i}$ is the height of the $i$ th isopycnal and $V=L_{x} L_{y} H$ is the volume of the basin with $H=\sum_{i=1}^{3} H_{i}$. We computed $P(t)$ for both F11 and CBD solutions, and compared them against those for the coarseand high-resolution solutions. Total available potential energy is an essential global characteristic of the solutions, and here we seek the manifestation of the intrinsic large-scale low-frequency variabilities (LFVs), which are interrelated to other large-scale flow features, such as the eastward jet extension and its adjacent recirculation zones 

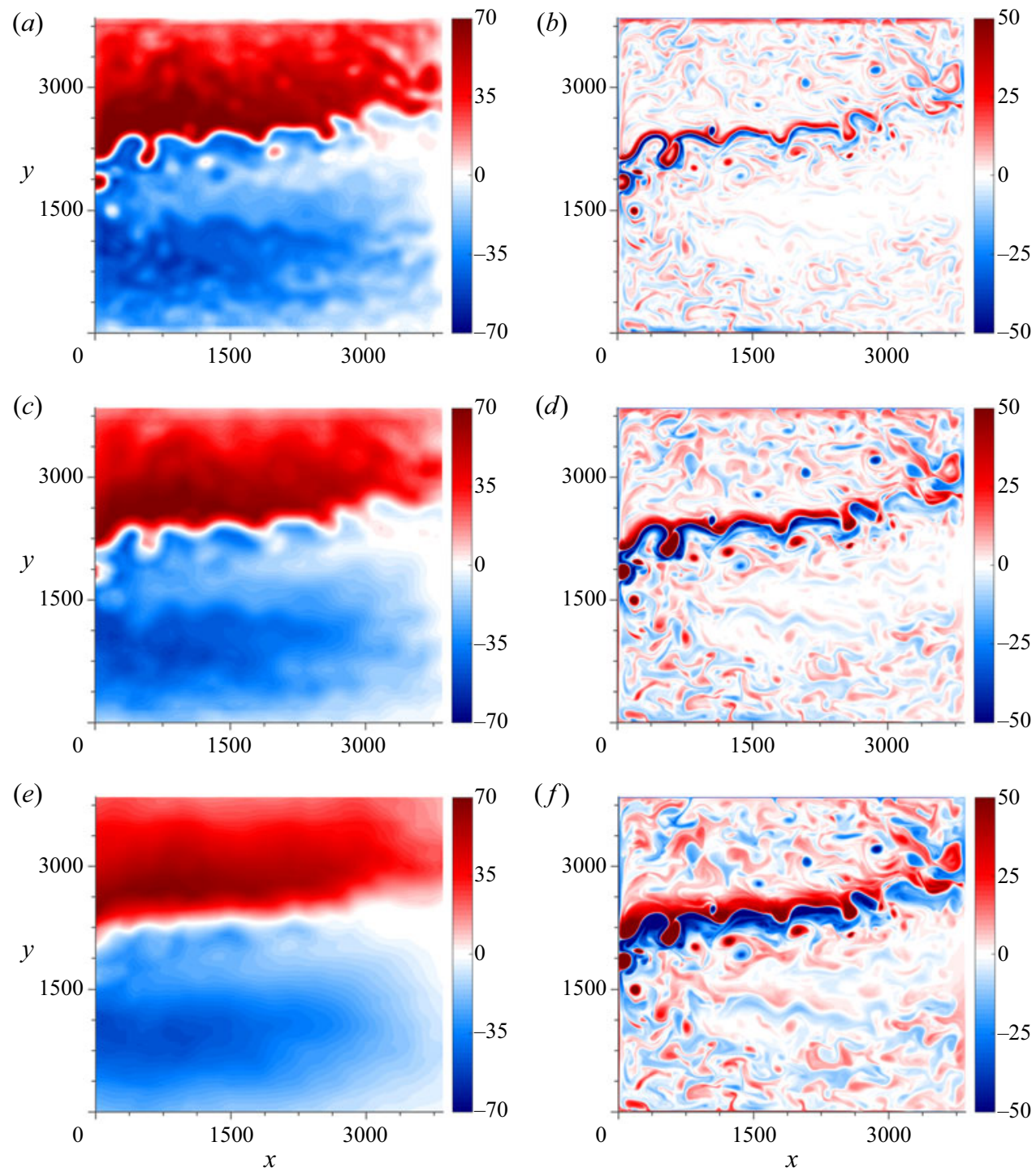

Figure 14. Effects of filter size on the large-scale and eddy fields. Snapshots of the top-layer large-scale and eddy PVAs, $\bar{q}_{1}$ and $q_{1}^{\prime}$, obtained using moving-average filtering with various filter sizes. Panels $(a, c, e) /$ $(b, d, f)$ show large-scale/eddy patterns for the $(a, b) \mathrm{F} 11,(c, d) \mathrm{F} 20$ and $(e, f) \mathrm{F} 40$ filters. The colour scales are in non-dimensional units.

(Berloff, Hogg \& Dewar 2007b). Berloff et al. (2007a) also showed that such LFVs feedback on the atmosphere in ocean-atmosphere coupled models and, therefore, are of significant dynamical importance. At high spatial resolutions, the reference flow regime possesses broadband energy modulations around 17 years variability, i.e. at a frequency value of 0.06 years $^{-1}$, whereas the coarse-resolution solution fails to simulate this, because of the poorly resolved eddies (figure 13). Therefore, feeding in dynamically consistent eddy statistics is necessary for strengthening the eastward jet and the associated LFV. The Fourier spectra of the total potential energy (figure 13) for CBD suggests the presence of pronounced broadband LFV, as contrasted to F11, with an order of magnitude difference 
between the corresponding spectral powers. This is a remarkable characteristic of CBD, as earlier, Ryzhov et al. (2020) showed that imbuing LFVs in the augmented solutions by merely feeding the eddies from fixed-size moving average filtering is hardly possible (even for larger filter sizes, e.g. F20). Reconstruction of the LFV is a significant advantage of $\mathrm{CBD}$, and it additionally proves that the decomposed eddy fields interact with the coarse-resolution solutions in a more dynamically correct sense, so that these solutions closer resemble the high-resolution reference flow regime.

Overall, our study confirmed that augmentation of the model restored the key large-scale flow features (e.g. the eastward jet) and the important LFV in the coarse-resolution solutions. This was made possible by plainly supplying the eddy fields extracted from a high-resolution solution. But this requires a more objective and precise scale separation method, such as those based on actual operating correlation length scales, their anisotropy and their orientation. The success of CBD encourages us to look for efficient statistical emulators to model the corresponding eddy fields and supply them instead of the true eddy fields, but this is left for future work.

\section{Conclusions and discussion}

This study aims to develop methods and approaches for exploring the properties of oceanic mesoscale eddies, study their dynamical feedbacks on the underlying large-scale flow and use them to augment non-eddy-resolving ocean model solutions. For this purpose, we used an eddy-resolving, double-gyre, classical quasigeostrophic model solution as an idealized representation of the North Atlantic or North Pacific ocean circulations. Defining and understanding fundamental statistical and dynamical properties of the oceanic mesoscale eddies is important for their parametrization in the non-eddy-resolving and eddy-permitting oceanic general circulation models. In this regard, dealing with the correct and relevant eddy statistics is crucial. The existing flow decomposition (i.e. scale separation) methods, such as the Reynolds decomposition, moving average, etc., which filter out an eddy component from the flow field, proved their usefulness but also have significant shortcomings. While the classical Reynolds decomposition does not incorporate any scale information, the moving-average decomposition imposes a filter size that is fixed across the domain and based on some scale assumption, such as assuming that the eddies operate on the first baroclinic Rossby deformation radius everywhere. Such an assumption is not necessarily accurate given the intrinsically multiscale nature of the eddies and of their underlying large-scale flow field. Another common strategy is to choose the filter size based on the resolution of the coarse-grid model, which needs eddy parametrizations. However, a nominally resolved flow feature in a dynamical model is not necessarily dynamically resolved with needed accuracy, and there is a broad 'grey zone' of partially resolved scales that further complicates the choice of filter size. This motivated us to explore a range of other filtering possibilities and look at the underlying differences to develop a better understanding of the eddy scales. Here, we have proposed a correlation-based decomposition (CBD) method which decomposes the flow using the concept of a differential filter size varying across the domain and based on the local correlation length scale diagnosed from the spatial correlation maps. In other words, instead of imposing an a priori assumption, we let the flow correlation statistics make its own decision on the filtering scale, which is now allowed to vary across the domain and be independent of the Rossby radius. We extracted the spatially varying length scale statistics using a Gaussian-shape fit to the local correlation maps, which additionally infers anisotropy and orientation from the correlation maps. Later on, we use a map of Gaussian kernels with the inferred parameters and filter out the reference flow field. In practice, 


\section{Correlation-based decomposition and eddy forcing analysis}

the correlation length scales are to be diagnosed from the available observations and/or realistic model-generated solutions.

We applied the CBD method to the potential vorticity anomaly (PVA) datasets and inverted them to get the corresponding streamfunction $(\psi)$ fields. Encouraging structural differences were found between the CBD decomposed eddy fields and the original flow features, as the former clearly absorbed the small-scale content of the latter. Furthermore, for comparison purpose, we considered uniform moving-average and spectral filtering methods. For the moving-average, we considered three filter sizes (F11, F20 and F40), where the smallest one (F11) was nearest to the mean (and median) length scale of the spatial correlation map. The eddy fields from both F11 and CBD were found to be reasonable but notably different. On the other hand, the results from the spectral filtering, with a cut-off wavenumber equivalent to the F11 filter size, was found to be heavily noisy due to the sharp-gradient regions in the flow that make the eddies spectrally non-local. Therefore, this method was discarded from further analyses, with the results briefly presented as a practical warning for other researchers.

In the second half of the paper, on the basis of the novel flow decomposition, we studied the causality between the reference eddy forcing and the induced large-scale flow. This causality - referred to as the eddy backscatter mechanism - was analysed by using the time-lagged product integral correlation time series. Here, the product integral correlation is the spatial diagnostic characteristic, which proved to be useful for the analyses by illuminating the dynamical importance of the transient eddy forcing. Both CBD- and F11-decomposed fields showed active eddy backscatter, which is the correlation of the large-scale PV anomalies with the transient eddy forcing, and captured its response time scale of approximately a week. However, CBD showed more efficient backscatter in the eastward jet region, where the eddies are most active. These results suggest that positive/negative transient eddy forcing enhances positive/negative PV anomalies, which eventually results in the maintenance of the eastward jet. We argue that the backscatter properties of the mesoscale eddies studied here should be taken into account by eddy parametrization schemes, e.g. as in the work of Berloff (2018), where a similar effect was achieved by amplifying the tendency term.

The other moving-average filters (F20 and F40) underestimated the correlation magnitude and overestimated the backscatter time scale, both being proportional to the filter size. This suggests that the CBD approach is intrinsically more robust, as the optimal filter size is chosen automatically. It can also have additional advantages for the unstructured grids, where moving-average filtering will have additional problems. Finally, we proved the dynamical importance of the CBD-produced eddy fields by feeding them to a coarse-resolution model solution. The results showed that the augmented solution recovers the otherwise missing eastward jet extension, the eddy variability around it and the intrinsic interdecadal low-frequency variabilities. The revival of the robust low-frequency variabilities points to a much superior dynamical correction produced by the CBD eddy fields compared with the fixed-size moving-average filters, which require additional information from the high-resolution solutions even for the most intuitive filter sizes (Ryzhov et al. 2020). This motivates us to explore in future studies statistical emulation methods (Kondrashov et al. 2018; Agarwal et al. 2021) for building low-order data-driven eddy emulators for use in coarse-resolution model augmentation.

On the downside, we note that CBD filters are computationally more expensive due to the involved Gaussian shape fitting for each grid node. For serial implementation, the Gaussian parameter computation takes longer time than the CBD decomposition of one flow snapshot, but both processes can be parallelized. Another shortcoming of CBD is that the length scale maps would be different for different variables in the case of 
the primitive equations, and determining the most relevant one would be difficult and would require more analyses. Subsequently, it remains to be seen how accurate CBD is near the continental boundaries in realistic solutions of comprehensive general circulation model solutions, as we only concentrated on reproducing the jet features in the current implementation. The boundary regions generally possess more anisotropic correlations, and, hence, $\mathrm{CBD}$ can be more effective in these regions than other isotropic and fixed-size filters.

The presented results can be extended along the following lines. The first and straightforward path is to extend our analyses for higher spatial resolutions and solutions of comprehensive general circulation models with realistic continental boundaries. Second, it is worthwhile to advance the CBD further using time-lagged correlation to provide both the spatial and temporal dependence of the length scales (Kondrashov, Ryzhov \& Berloff 2020). A scale separation based on such non-stationary, spatially varying length scales can be more consistent dynamically. Next, the statistical properties of the eddy forcing studied in this paper can be effectively used for developing statistically and dynamically consistent, data-driven eddy emulators for use in eddy parametrizations. Subsequently, the eddy emulators can be more efficiently used to augment low-resolution models, similar to what was achieved recently by Ryzhov et al. (2020) for a simple moving-average flow decomposition. Here, we focused on finding novel ways of defining eddies, studying their feedbacks on the induced large-scale flow structures and their possible augmentation skills, and these aspects provide the basis for further advances. Finally, looking for connections between local convolution-based filters and dynamical filtering (e.g. Berloff et al. 2021) is also a matter for future research.

Acknowledgements. The authors would like to thank Dr P. Dueben for many useful discussions and the Research Computing Service (RCS) team of Imperial College London for the help and assistance with HPC. The authors are also thankful to all the anonymous reviewers for their feedback and suggestions, which improved the manuscript significantly.

Funding. N.A. is thankful to Mathematics of Planet Earth Centre for Doctoral Training (MPE CDT) for providing the financial and technical support to carry out this research. P.B. was supported by the NERC grants NE/R011567/1 and NE/T002220/1, the Leverhulme grant RPG-2019-024, and Moscow Center of Fundamental and Applied Mathematics (supported by the Agreement 075-15-2019-1624 with the Ministry of Education and Science of the Russian Federation). E.R. is supported by NERC grant NE/R011567/1 and Royal Society Exchange Grant IEC/R2/181033; D.K. was supported by the National Science Foundation grant OCE-1658357. CBD analysis in $\S 3.1$ was supported by the Russian Science Foundation (Grant No. 18-12-00231). E.R. was partly supported by the Russian Science Foundation (Project 19-17-00006) in formulating the general concept of the varying filter-size flow decomposition based on the local correlation maps and clustering in baroclinic ocean flows. E.R. also thanks the 'Imperial CoA NERC' Covid-extension grant. All the source codes and datasets are available upon request.

Declaration of interests. The authors report no conflict of interests.

Author ORCIDs.

(1) N. Agarwal https://orcid.org/0000-0002-4095-5827;

E.A. Ryzhov https://orcid.org/0000-0002-2677-5723;

D. Kondrashov https://orcid.org/0000-0002-3471-7275;

D. Berloff https://orcid.org/0000-0001-9143-5955.

\section{Appendix A. Moving-average filtering}

This is the most basic type of filtering, in which a kernel of appropriate geometry and size is convoluted with the flow variable to obtain the large-scale flow field, and the eddy field is obtained as the difference. Mathematically, if $\phi$ is a space-time dependent variable, then 



Figure 15. Same as in figure 14 but for the transport streamfunctions $\bar{\psi}_{1}$ and $\psi_{1}^{\prime}$.

$$
\begin{gathered}
\bar{\phi}(x ; t)=\int \phi(\xi ; t) G(x-\xi ; t) \mathrm{d} \xi, \\
\phi^{\prime}(x ; t)=\phi(x ; t)-\bar{\phi}(x ; t),
\end{gathered}
$$

where $G$ is a circular and normalized top-hat filtering kernel, whose size (e.g. radius) depends on the cut-off length scale; and the integration is applied over the whole domain. In geophysical fluids, the cut-off length scale can be related to the first baroclinic Rossby deformation radius $R d_{1}$ and, as described in the text, we used three values of the cut-off length scale: 11, 20 and 40 grid intervals (hereafter, referred as F11, F20 and F40 filters), which correspond to $77.5 \mathrm{~km}, 150 \mathrm{~km}$ and $300 \mathrm{~km}$, respectively (in terms of $R d_{1}$ this corresponds to approximately $2 R d_{1}, 3.75 R d_{1}$ and $7.5 R d_{1}$ ). Near the boundaries the filtering is done by convoluting with the partial (within the domain) circles. 

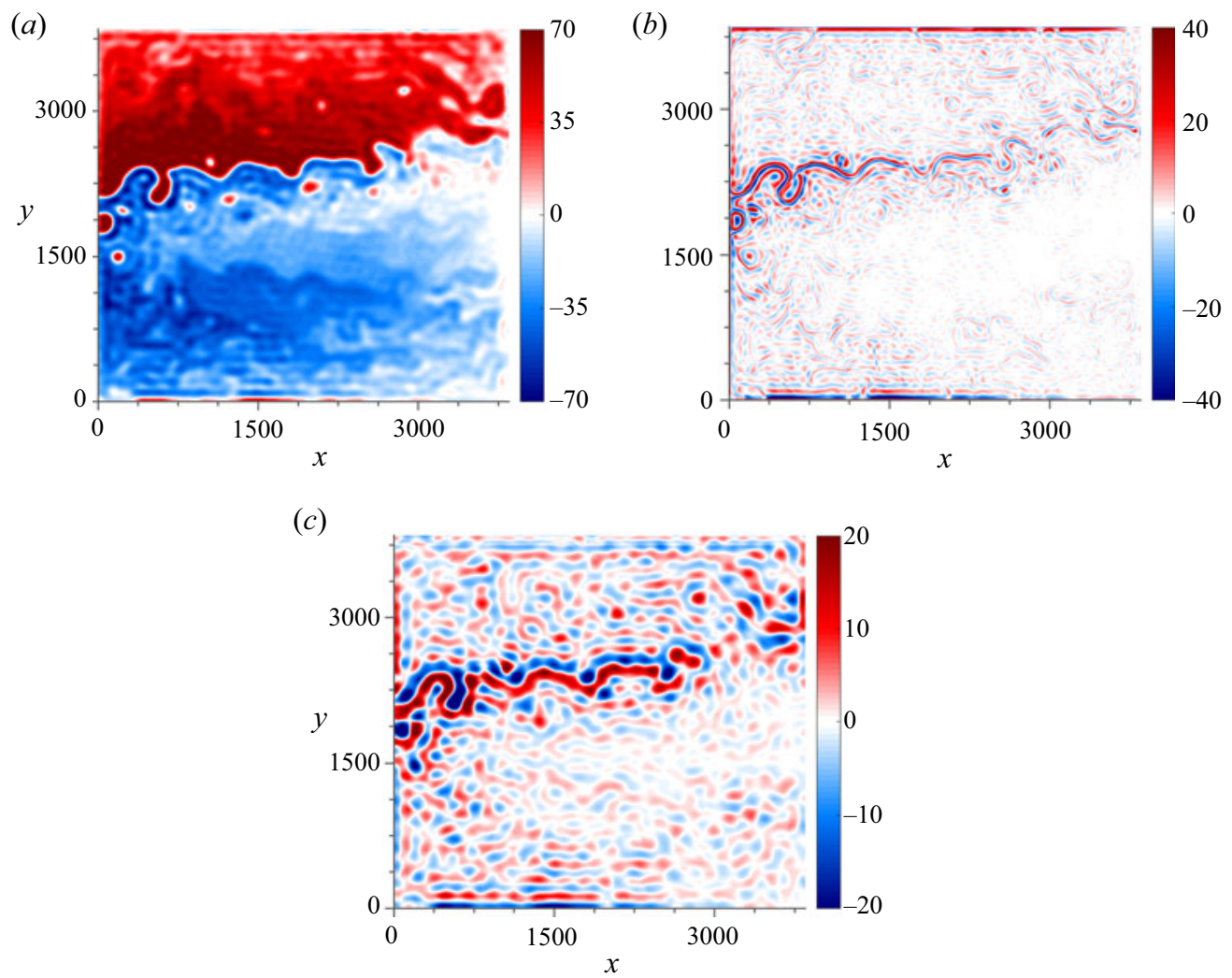

Figure 16. Spectral filtering results: (a) $\bar{q}_{1}$ and (b) $q_{1}^{\prime}$, obtained using a cut-off wavenumber equivalent to F11 moving-average filter size. (c) Difference between $q_{1}^{\prime}$ from spectral and moving-average filterings shows non-locality of the spectral filtering method. The noise in the decomposed fields is due to the problems of the spectral filtering method on the high gradients/discontinuities in the double-gyre flow fields.

The filtering is applied layer-wise and to $q$, whereas $\psi$ is obtained by inverting $((2.4 a)-(2.4 c))$. Many eddy features can be seen in the eddy fields, and their number and amplitude increase with the filter size; at the same time the large-scale fields become smoother (figures 14 and 15).

In the context of geophysical fluids, moving-average filtering has problems, and the main one is to choose the cut-off scale. Inhomogeneous, anisotropic and confined turbulence, in addition to the baroclinic Rossby deformation radii, has other relevant and geographically varying length scales characterizing the eddies. Here, we expect smaller and more vigorous eddies to populate the eastward jet region, and this is likely to be expressed by shorter spatial correlations. However, much longer correlations are expected in the interior gyres, which are mostly populated by well-seen large-scale transient planetary waves distorted by the circulation in the gyres.

\section{Appendix B. Spectral filtering}

Spectral filtering can be described as a spectral-domain analogy of the physical-space moving-average; its high-pass content constitutes the eddies. A spectral filter is local in the Fourier space but non-local in the physical space and, intuitively, it is expected to be optimal in the situation of homogeneous turbulence populated by plane waves. 


\section{Correlation-based decomposition and eddy forcing analysis}

We carried out the following: (i) applied Fourier transform to the spatial field; (ii) set to zero the spectral coefficients for wavenumbers higher than a certain threshold; (iii) performed the inverse Fourier transform of the outcome. The algorithm was implemented on the double-periodic domain - obtained by copying the mirror image of the data in both $x$ and $y$ directions - to deal with the boundary effects. We applied this filtering to PVA fields using a cut-off wavenumber $\left(k_{c}\right)$ equivalent to the F11 filter size, i.e. $k_{c}=2 \pi / 22 \Delta x$ (figure $16 a, b$ ).

By comparing with figure 14(a,b), it can be concluded that both flow components from the spectral filtering are spuriously patterned and noisier than the moving-average ones, especially the eddy field. The reasons behind these discrepancies are the large-gradient regions, such as the eastward jet and numerous coherent vortices. They act like step-functions which are composed of many non-locally distributed harmonics in the Fourier domain. Therefore, the fields corresponding to the residual power in high/low wavenumbers appear in small-/large-scale flow components in the form of noisy structures (figure 16c).

Therefore, we conclude that spectral filtering is not optimal for decomposition of our flow solutions. Note that alternatives such as wavelet filtering and short-term Fourier transform can be used to overcome the high-gradient and boundary conditions issues, but such methods have additional technical difficulty in terms of dealing with the size and filtered content of the wavelets/window and, therefore, do not fit into the scope of this study.

\section{REFERENCES}

Agarwal, N., Kondrashov, D., Dueben, P., Ryzhov, E. \& Berloff, P. 2021 A comparison of data-driven approaches to build low-dimensional ocean models. J. Adv. Model. Earth Syst. (submitted).

Aluie, H. \& EYINK, G. 2009 Localness of energy cascade in hydrodynamic turbulence. II. Sharp spectral filter. Phys. Fluids 21 (11), 115108.

Aluie, H., Hecht, M. \& Vallis, G. 2018 Mapping the energy cascade in the north atlantic ocean: the coarse-graining approach. J. Phys. Oceanogr. 48 (2), 225-244.

ARAKAwA, A. 1966 Computational design for long-term numerical integration of the equations of fluid motion: two-dimensional incompressible flow. Part I. J. Comput. Phys. 1 (1), 119-143.

Berloff, P. 2005 On dynamically consistent eddy fluxes. Dyn. Atmos. Oceans 38 (3-4), 123-146.

BERLOFF, P. 2015 Dynamically consistent parameterization of mesoscale eddies. Part I: simple model. Ocean Model. 87, 1-19.

BERLOFF, P. 2018 Dynamically consistent parameterization of mesoscale eddies. Part III: deterministic approach. Ocean Model. 127, 1-15.

Berloff, P., Dewar, W., Kravtsov, S. \& MCWilliams, J. $2007 a$ Ocean eddy dynamics in a coupled ocean-atmosphere model. J. Phys. Oceanogr. 37 (5), 1103-1121.

Berloff, P., HoGg, A.M.C. \& DewAR, W. $2007 b$ The turbulent oscillator: a mechanism of low-frequency variability of the wind-driven ocean gyres. J. Phys. Oceanogr. 37 (9), 2363-2386.

Berloff, P., Ryzhov, E. \& Shevchenko, I. 2021 On dynamically unresolved oceanic mesoscale motions. J. Fluid Mech. 920, A41.

BERLOFF, P.S. \& MCWILliams, J.C. 1999 Quasigeostrophic dynamics of the western boundary current. J. Phys. Oceanogr. 29 (10), 2607-2634.

Bolton, T. \& ZannA, L. 2019 Applications of deep learning to ocean data inference and subgrid parameterization. J. Adv. Model. Earth Syst. 11 (1), 376-399.

Crommelin, D. \& VANDEN-Eijnden, E. 2008 Subgrid-scale parameterization with conditional markov chains. J. Atmos. Sci. 65 (8), 2661-2675.

DUAN, J. \& NADIGA, B. 2007 Stochastic parameterization for large eddy simulation of geophysical flows. Proc. Am. Math. Soc. 135 (4), 1187-1196.

FREDERIKSEN, J. 1999 Subgrid-scale parameterizations of eddy-topographic force, eddy viscosity, and stochastic backscatter for flow over topography. J. Atmos. Sci. 56 (11), 1481-1494.

Frederiksen, J. \& DAVIES, A. 1997 Eddy viscosity and stochastic backscatter parameterizations on the sphere for atmospheric circulation models. J. Atmos. Sci. 54 (20), 2475-2492. 


\section{N. Agarwal, E.A. Ryzhov, D. Kondrashov and P. Berloff}

Gent, P. \& MCWilliams, J. 1990 Isopycnal mixing in ocean circulation models. J. Phys. Oceanogr. 20 (1), 150-155.

Gent, P., Willebrand, J., McDougall, T. \& McWilliams, J. 1995 Parameterizing eddy-induced tracer transports in ocean circulation models. J. Phys. Oceanogr. 25 (4), 463-474.

Grooms, I. \& MAJdA, A. 2013 Efficient stochastic superparameterization for geophysical turbulence. Proc. Natl Acad. Sci. 110 (12), 4464-4469.

Gugole, F. \& FRANZKE, C.L.E. 2020 Spatial covariance modeling for stochastic subgrid-scale parameterizations using dynamic mode decomposition. J. Adv. Model. Earth Syst. 12 (8), e2020MS002115.

HewitT, H.T., et al. 2020 Resolving and parameterising the ocean mesoscale in earth system models. Curr. Clim. Change Rep. 6, 137-152.

Holloway, G. 1987 Systematic forcing of large-scale geophysical flows by eddy-topography interaction. J. Fluid Mech. 184, 463-476.

Karabasov, S., Berloff, P. \& Goloviznin, V. 2009 Cabaret in the ocean gyres. Ocean Model. 30 (2-3), $155-168$.

Kitsios, V., Frederiksen, J. \& Zidikheri, M. 2012 Subgrid parameterisation of the eddy-meanfield interactions in a baroclinic quasi-geostrophic ocean. ANZIAM J. 54, 459-475.

KondRASHOV, D. \& BERLOFF, P. 2015 Stochastic modeling of decadal variability in ocean gyres. Geophys. Res. Lett. 42 (5), 1543-1553.

Kondrashov, D., Chekroun, M. \& Berloff, P. 2018 Multiscale stuart-landau emulators: application to wind-driven ocean gyres. Fluids 3 (1), 21.

Kondrashov, D., RYZHov, E. \& BERLOFF, P. 2020 Data-adaptive harmonic analysis of oceanic waves and turbulent flows. Chaos 30, 061105.

Kraichnan, R. 1976 Eddy viscosity in two and three dimensions. J. Atmos. Sci. 33 (8), 1521-1536.

LEITH, C. 1990 Stochastic backscatter in a subgrid-scale model: plane shear mixing layer. Phys. Fluids A 2 (3), 297-299.

LEONARD, A. 1975 Energy cascade in large-eddy simulations of turbulent fluid flows. In Advances in Geophysics (ed. F.N. Frenkiel \& R.E. Munn), vol. 18, pp. 237-248. Elsevier.

ManA, P. \& ZANnA, L. 2014 Toward a stochastic parameterization of ocean mesoscale eddies. Ocean Model. 79, 1-20.

NAdigA, B. 2008 Orientation of eddy fluxes in geostrophic turbulence. Phil. Trans. R. Soc. A 366 (1875), 2489-2508.

NADIGA, B.T. \& LivesCU, D. 2007 Instability of the perfect subgrid model in implicit-filtering large eddy simulation of geostrophic turbulence. Phys. Rev. E 75 (4), 046303.

Percival, D.B., et al. 1993 Spectral Analysis for Physical Applications. Cambridge University Press.

Piomelli, U., CAвот, W., Moin, P. \& LeE, S. 1991 Subgrid-scale backscatter in turbulent and transitional flows. Phys. Fluids A 3 (7), 1766-1771.

Ryzhov, E., Kondrashov, D., Agarwal, N. \& Berloff, P. 2019 On data-driven augmentation of low-resolution ocean model dynamics. Ocean Model. 142, 101464.

Ryzhov, E., Kondrashov, D., Agarwal, N., McWilliams, J. \& Berloff, P. 2020 On data-driven induction of the low-frequency variability in a coarse-resolution ocean model. Ocean Model. 153, 101664.

SAgAut, P. 2006 Large eddy Simulation for Incompressible Flows: An Introduction. Springer Science \& Business Media.

Shevchenko, I. \& Berloff, P. 2015 Multi-layer quasi-geostrophic ocean dynamics in eddy-resolving regimes. Ocean Model. 94, 1-14.

SheVCHENKO, I. \& BERLOFF, P. 2016 Eddy backscatter and counter-rotating gyre anomalies of midlatitude ocean dynamics. Fluids 1 (3), 28.

Vreman, B., Geurts, B. \& Kuerten, H. 1994 Realizability conditions for the turbulent stress tensor in large-eddy simulation. J. Fluid Mech. 278, 351-362.

Zanna, L., Mana, P., Anstey, J., David, T. \& Bolton, T. 2017 Scale-aware deterministic and stochastic parametrizations of eddy-mean flow interaction. Ocean Model. 111, 66-80. 